DESY 09-133

SFB/CPP-09-80

LTH 840

September 2009

\title{
On non-singlet physical evolution kernels and large- $x$ coefficient functions in perturbative QCD
}

\author{
S. Moch ${ }^{a}$ and A. Vogt ${ }^{b}$ \\ ${ }^{a}$ Deutsches Elektronensynchrotron DESY \\ Platanenallee 6, D-15738 Zeuthen, Germany \\ ${ }^{b}$ Department of Mathematical Sciences, University of Liverpool \\ Liverpool L69 3BX, United Kingdom
}

\begin{abstract}
We study the large- $x$ behaviour of the physical evolution kernels for flavour non-singlet observables in deep-inelastic scattering, where $x$ is the Bjorken variable, semi-inclusive $e^{+} e^{-}$annihilation and Drell-Yan lepton-pair production. Unlike the corresponding $\overline{\mathrm{MS}}$-scheme coefficient functions, all these kernels show a single-logarithmic large- $x$ enhancement at all orders in $1-x$. We conjecture that this universal behaviour, established by Feynman-diagram calculations up to the fourth order, holds at all orders in the strong coupling constant $\alpha_{\mathrm{s}}$. The resulting predictions are presented for the highest $\ln ^{n}(1-x)$ contributions to the higher-order coefficient functions. In Mellin- $N$ space these predictions take the form of an exponentiation which, however, appears to be less powerful than the well-known soft-gluon exponentiation of the leading $(1-x)^{-1} \ln ^{n}(1-x)$ terms. In particular in deep-inelastic scattering the $1 / N$ corrections are non-negligible for all practically relevant $N$.
\end{abstract}




\section{Introduction}

Disregarding power corrections, hard hadron processes are described in perturbative QCD in terms of process-dependent short-distance coefficient functions (mass-factorized partonic cross sections) and universal space- and timelike parton densities including non-perturbative long-distance effects. The separation between the coefficient functions and the parton densities and the splitting functions governing their scale dependence is, of course, not unique beyond the leading order (LO) in perturbative QCD. It is usual to perform this separation in the modified [1] minimal subtraction [2] scheme, $\overline{\mathrm{MS}}$, see also Ref. [3], of dimensional regularization [4], the standard framework for higher-order diagrammatic calculations in quantum field theory.

While this scheme does not provide a physical definition of the parton densities, it does lead to a stable (order-independent) functional form of the dominant diagonal (quark-quark and gluongluon) splitting functions in the limit of large momentum fractions $x$ [5-10]. This feature assists a stable evolution of the parton densities over a wide range of scales as required, e.g., for LHC predictions based on data from fixed-target and HERA experiments.

The coefficient functions, on the other hand, receive double logarithmic large- $x$ enhancements in the $\overline{\mathrm{MS}}$ scheme, i.e., terms up to $(1-x)^{-1+k} \ln ^{2 n-a}(1-x)$ occur, for all $k$, at the $n$-th order of the strong coupling constant $\alpha_{\mathrm{s}}$ (the offset $a \geq 1$ depends on the observable and the power $k$ in the expansion in $1-x)$. The highest leading $(k=0)$ logarithms can be resummed by the soft-gluon exponentiation [11] which is now known to the next-to-next-to-next-to-leading logarithmic ( $\left.{ }^{3} \mathrm{LL}\right)$ accuracy for inclusive deep-inelastic lepton-proton scattering (DIS), $l p \rightarrow l+X$ [12], Drell-Yan (DY) lepton-pair production and Higgs production in proton-proton collisions [13-15], and semiinclusive electron-positron annihilation (SIA), $e^{+} e^{-} \rightarrow h+X(h=\pi, K, \ldots)[16,17]$. On the other hand, recent studies of the subleading $k=1$ logarithms $[18,19]$ have not led to similarly systematic predictions for higher-order coefficient functions yet.

An alternative description of hard processes can be obtained by eliminating the parton densities, leading to physical evolution kernels (also called physical anomalous dimensions) for the scale dependence of observables [3], see also Ref. [20]. This is especially simple for the flavour non-singlet quantities dominating the large- $x$ limits of the semi-leptonic DIS, SIA and DY processes mentioned above. The direct relation between different processes via the universal parton densities is absent in this approach, but the soft-gluon exponentiation [11] guarantees an only single logarithmic $k=0$ higher-order large- $x$ enhancement [21], see also Refs. [22].

Using the coefficient-function results of Refs. [23-32] for the above processes, one finds that the corresponding non-singlet physical kernels exhibit only a single logarithmic enhancement for all values of $k$ at least to the next-to-next-to-leading or next-to-next-to-next-to-leading order (NNLO or $\mathrm{N}^{3} \mathrm{LO}$ ) in the expansion in $\alpha_{\mathrm{s}}$. We are thus led to the rather obvious conjecture that this behaviour, already established to order $\alpha_{\mathrm{s}}^{4}$ in DIS, persists to all orders in $\alpha_{\mathrm{s}}$. The required cancellation of double logarithms in the physical kernels then implies exponentiations also of terms with $k \geq 1$, yielding explicit all-order predictions for the highest logarithms in the respective quark coefficient functions. In the rest of this article we derive and discuss these predictions, emphasizing the subleading $k=1$ logarithms. Especially for this case a formal proof of the exponentiation may be expected in the near future from the new path-integral approach of Ref. [19]. 


\section{Physical evolution kernels for non-singlet observables}

We start by recalling the construction and fixed-order properties of the physical evolution kernels. We first consider the DIS structure functions (see Ref. [33] for a general overview)

$$
\mathcal{F}_{1}=2 F_{1, \mathrm{~ns}}, \quad \mathcal{F}_{2}=\frac{1}{x} F_{2, \mathrm{~ns}}, \quad \mathcal{F}_{3}=F_{3}{ }^{\mathrm{v}+\overline{\mathrm{v}}} .
$$

The longitudinal structure function $F_{L}=F_{2}-2 x F_{1}$ has been addressed already in Ref. [34]. Disregarding terms suppressed by powers of $1 / Q^{2}$, the non-singlet quantities (2.1) are given by

$$
\mathcal{F}_{a}\left(x, Q^{2}\right)=\left[C_{a}\left(Q^{2}\right) \otimes q_{a, \mathrm{~ns}}\left(Q^{2}\right)\right](x)=\sum_{l=0} a_{s}^{l}\left(Q^{2}\right)\left[c_{a, l} \otimes q_{a, \mathrm{~ns}}\left(Q^{2}\right)\right](x) .
$$

As usual $x$ is the Bjorken variable, and $Q^{2}=-q^{2}$ the negative squared four-momentum of the exchanged gauge boson. $c_{a, l}$ represents the $l$-loop non-singlet coefficient function for $\mathcal{F}_{a}$ with $c_{a, 0}(x)=\delta(1-x)$. The exact three-loop results $c_{a, 3}(x)$ for the structure functions (2.1) have been computed in Refs. [26-28]. Beyond this order only the $C_{F} n_{f}^{l-1}$ leading- $n_{f}$ terms are exactly known $[35,36]$. Furthermore $q_{a, \text { ns }}$ denotes the corresponding combination of the quark densities (including electroweak charge factors), and $\otimes$ stands for the standard Mellin convolution, given by

$$
[a \otimes b](x)=\int_{x}^{1} \frac{d y}{y} a(y) b\left(\frac{x}{y}\right)
$$

for two regular functions and Eq. (3.4) of Ref. [21] if a +-distribution is involved. The renormalization and factorization scales $\mu_{\mathrm{r}}$ and $\mu_{\mathrm{f}}$ have been set to the physical hard scale $Q$ in Eq. (2.2).

The scale dependence of the running coupling of QCD, in this article normalized as

$$
a_{s} \equiv \frac{\alpha_{s}}{4 \pi}
$$

is governed by

$$
\frac{d a_{s}}{d \ln Q^{2}}=\beta\left(a_{s}\right)=-\sum_{l=0} a_{s}^{l+2} \beta_{l} .
$$

Besides the scheme-independent $\beta_{0}=11 / 3 C_{A}-2 / 3 n_{f}$ [37] (with $C_{A}=N_{\text {colours }}=3$ in QCD) and $\beta_{1}$ [38], also the coefficients $\beta_{2}$ and $\beta_{3}$ have been computed $[39,40]$ in the $\overline{\mathrm{MS}}$ renormalization scheme adopted throughout this study. All these four coefficients are required for calculations including the $\mathrm{N}^{3} \mathrm{LO}$ quantities $c_{a, 3}$. Here and below $n_{f}$ denotes the number of effectively massless flavours (mass effects are not considered in this article). Finally the evolution equations for the quark densities in Eq. (2.2) read

$$
\begin{aligned}
\frac{d}{d \ln Q^{2}} q_{a, \mathrm{~ns}}\left(x, Q^{2}\right) & =\left[P_{a}\left(Q^{2}\right) \otimes q_{a, \mathrm{~ns}}\left(Q^{2}\right)\right](x) \\
& =\sum_{l=0} a_{s}^{l+1}\left[P_{a, l} \otimes q_{a, \mathrm{~ns}}\left(Q^{2}\right)\right](x) .
\end{aligned}
$$

As the coefficient functions $c_{a, l}$, the $(l+1)$-loop splitting functions $P_{a, l}$ depend only on $x$ for the above choice of $\mu_{\mathrm{r}}$ and $\mu_{\mathrm{f}}$. All three independent third-order (NNLO) non-singlet splitting functions $P_{a, 2}(x)$ are known from Ref. [6]. 
The convolutions in Eqs. (2.2) and (2.5) correspond to simple products of the respective Mellin transforms given by

$$
a^{N}=\int_{0}^{1} d x x^{N-1} a(x)
$$

for regular functions such as $q_{a, \mathrm{~ns}}$ and

$$
a^{N}=\int_{0}^{1} d x\left(x^{N-1}-1\right) a(x)_{+}
$$

for +-distributions such as the leading large- $x$ contributions to $c_{a, l}(x)$. Hence calculations involving multiple convolutions as, e.g., in Eqs. (2.9) below are best carried out in $N$-space where the coefficient functions and splitting functions are expressed in terms of harmonic sums [41]. We mainly use FORM [42] and TFORM [43] to manipulate such expressions, to transform them back to the $x$-space harmonic polylogarithms [44], see also Ref. [24], and to extract large- $x$ coefficients from the results.

It is convenient, both phenomenologically - for instance for determinations $\alpha_{\mathrm{S}}$ - and theoretically, to express the scaling violations of non-singlet observables in terms of these observables themselves. This explicitly eliminates any dependence on the factorization scheme and the associated scale $\mu_{\mathrm{f}}$, and avoids the non-negligible dependence of the $\overline{\mathrm{MS}}$-scheme initial distributions for $q_{a, \mathrm{~ns}}$ on the perturbative order. The corresponding physical evolution kernels $K_{a}$ can be derived for $\mu_{\mathrm{r}}^{2}=Q^{2}$ by differentiating Eq. (2.2) with respect to $Q^{2}$ by means of the respective evolution equations (2.4) and (2.5) for $a_{\mathrm{s}}$ and $q_{a, \mathrm{~ns}}$, and then using the inverse of Eq. (2.2) to eliminate $q_{a, \mathrm{~ns}}$ from the result. This procedure yields the evolution equations [21]

$$
\begin{aligned}
\frac{d}{d \ln Q^{2}} \mathcal{F}_{a} & =\left\{P_{a}\left(a_{\mathrm{s}}\right)+\beta\left(a_{\mathrm{s}}\right) \frac{d C_{a}\left(a_{\mathrm{s}}\right)}{d a_{\mathrm{s}}} \otimes C_{a}\left(a_{\mathrm{s}}\right)^{-1}\right\} \otimes \mathcal{F}_{a} \\
& \equiv K_{a} \otimes \mathcal{F}_{a} \equiv \sum_{l=0} a_{\mathrm{s}}^{l+1} K_{a, l} \otimes \mathcal{F}_{a} \\
& =\left\{a_{\mathrm{s}} P_{a, 0}+\sum_{l=1} a_{\mathrm{s}}^{l+1}\left(P_{a, l}-\sum_{k=0}^{l-1} \beta_{k} \tilde{c}_{a, l-k}\right)\right\} \otimes \mathcal{F}_{a} .
\end{aligned}
$$

Notice that in $N$-space the second term in the first line simply is $\beta\left(a_{\mathrm{s}}\right) d \ln C_{a}\left(a_{\mathrm{s}}\right) / d a_{\mathrm{s}}$. Up to $\mathrm{N}^{4} \mathrm{LO}$ (terms up to $l=4$ included in the sums) the expansion coefficients $\tilde{c}_{a, l}(x)$ in the last line read

$$
\begin{aligned}
\tilde{c}_{a, 1} & =c_{a, 1} \\
\tilde{c}_{a, 2} & =2 c_{a, 2}-c_{a, 1}^{\otimes 2} \\
\tilde{c}_{a, 3} & =3 c_{a, 3}-3 c_{a, 2} \otimes c_{a, 1}+c_{a, 1}^{\otimes 3} \\
\tilde{c}_{a, 4} & =4 c_{a, 4}-4 c_{a, 3} \otimes c_{a, 1}-2 c_{a, 2}^{\otimes 2}+4 c_{a, 2} \otimes c_{a, 1}^{\otimes 2}-c_{a, 1}^{\otimes 4}
\end{aligned}
$$

with $f^{\otimes 2} \equiv f \otimes f$ etc. The above expressions for $K_{a, l \geq 1}$ are valid for $\mu_{\mathrm{r}}=Q$, the explicit generalization to $\mu_{\mathrm{r}} \neq Q$ to this order can be found in Eq. (2.9) of Ref. [21].

The $\mathrm{N}^{3} \mathrm{LO}$ physical kernels for the structure functions (2.1) are not completely known at this point, as the four-loop splitting functions $P_{a, 3}(x)$ contributing to Eq. (2.8) have not been derived so 
far beyond the small leading- $n_{f}$ contribution [45]. Already the corresponding three-loop splitting functions $P_{a, 2}$, however, have only a small impact at $x>10^{-3}$, see Fig. 7 of Ref. [6]. Moreover, the dependence of the non-singlet splitting function on $N$ and on the specific quark combination is such that a single four-loop moment of any of them sets the scale for the $\mathrm{N}^{3} \mathrm{LO}$ contributions outside the small- $x$ region, cf. Fig. 1 of Ref. [6]. Such a calculation has been presented in Ref. [46], and the fourth-order correction is indeed found to be small. Hence a rough estimate of $P_{a, 3}(x)$, for instance via an $N$-space Padé estimate, is sufficient in Eq. (2.8) for all practical non-singlet analyses.

The expressions for the transverse, longitudinal and asymmetric fragmentation functions

$$
\mathcal{F}_{T}=F_{T, \mathrm{~ns}}^{h}, \quad \mathcal{F}_{L}=F_{L, \mathrm{~ns}}^{h}, \quad \mathcal{F}_{A}=F_{A}^{h}
$$

in semi-inclusive $e^{+} e^{-}$annihilation (see Ref. [33] for a general overview), $e^{+} e^{-} \rightarrow \gamma / Z \rightarrow h+X$, are completely analogous to those for the corresponding deep-inelastic structure functions. The scaling variable in Eq. (2.2) now reads $x=2 p q / Q^{2}$ where $q$ with $q^{2} \equiv Q^{2}>0$ is the momentum of the virtual gauge boson, and $p$ that of the identified hadron $h$. The second-order non-singlet coefficient functions $c_{a, 2}(x)$ for these cases have been calculated in Refs. [29], see also Ref. [9] where we have derived the corresponding timelike splitting functions $P_{a, 2}$ for the evolution of the non-singlet fragmentation densities $q_{a \text {,ns }}$ of the hadron $h$. In these cases we know the three-loop coefficient functions $c_{a, 3}$, beyond the leading large- $x$ terms of Refs. [16,17], only up some terms involving $\zeta_{2}=\pi^{2} / 6$, cf. the hadronic Higgs decay rate in Ref. [10]. These incomplete results have not been published. Their (complete) highest $\ln ^{n}(1-x)$ terms will be presented in the next section.

Finally we also consider the non-singlet quark-antiquark annihilation contribution to the total cross section for Drell-Yan lepton-pair production, $p p / p \bar{p} \rightarrow l^{+} l^{-}+X$,

$$
\mathcal{F}_{\mathrm{DY}}=\frac{1}{\sigma_{0}} \frac{d \sigma_{\mathrm{ns}}}{d Q^{2}} .
$$

In a rather schematic (but for our purpose sufficient) manner this quantity can be written as

$$
\mathcal{F}_{\mathrm{DY}}\left(x, Q^{2}\right)=\left[C_{\mathrm{DY}}\left(Q^{2}\right) \otimes q\left(Q^{2}\right) \otimes \bar{q}\left(Q^{2}\right)\right](x) .
$$

Here $Q^{2}>0$ denotes the squared invariant mass of the lepton pair, and the scaling variable is given by $x=Q^{2} / S$ where $S$ is the squared CMS energy of the proton-(anti-)proton initial state. As in the case of deep-inelastic scattering, $q\left(x, Q^{2}\right)$ represents the initial-state (spacelike) quark densities of the proton which evolve with the splitting functions of Ref. [34]. The non-singlet quark-antiquark coefficient function has a perturbative expansion analogous to Eq. (2.2) above, with $\sigma_{0}$ in Eq. (2.11) chosen such that also $c_{\mathrm{DY}, 0}(x)=\delta(1-x)$. The complete expressions for the NNLO contribution $c_{\mathrm{DY}, 2}(x)$ have been calculated in Refs. [31,32]. At $\mathrm{N}^{3} \mathrm{LO}$ only the leading large- $x$ terms, $(1-x)^{-1} \ln ^{n}(1-x)$ with $n=0, \ldots, 5$, are presently known from Ref. [13], see also Ref. [14].

The derivation of the physical evolution kernel for $\mathcal{F}_{\text {DY }}$ proceeds completely analogous to the paragraph of Eq. (2.8), with the non-singlet quark-quark splitting function occurring twice instead of once. As we will show in the next section, this modification is irrelevant for the purpose of this article, the determination of subleading large- $x$ / large- $N$ double logarithms in the higher-order coefficient functions for the quantities in Eqs. (2.1), (2.10) and (2.11). 


\section{Known large- $x$ logarithms at the second and third order}

We next need to address the expansions in powers of $\ln (1-x)$ of the known non-singlet splitting functions $[6,9,47,48]$ and coefficient functions [23-32] in the $\overline{\mathrm{MS}}$ scheme. The spacelike splitting functions (2.5) for all three types of non-singlet combinations of quark densities,

$$
q_{ \pm, \mathrm{ns}}^{(i k)}=q_{i} \pm \bar{q}_{i}-\left(q_{k} \pm \bar{q}_{k}\right), \quad q_{\mathrm{v}, \mathrm{ns}}=\sum_{r=1}^{n_{f}}\left(q_{r}-\bar{q}_{r}\right)
$$

are given by

$$
P_{a, l}(x)=\frac{A_{l+1}}{(1-x)_{+}}+\widetilde{B}_{l+1} \delta(1-x)+\widetilde{C}_{l+1} \ln (1-x)+o\left((1-x)^{k \geq 1} \ln ^{l}(1-x)\right) .
$$

The last term indicates that the $(l+1)$-loop splitting functions $P_{a, l}(x)$ receive contributions from terms no higher than $\ln ^{l}(1-x)$, and that these contributions occur at all orders in $(1-x)$ from the first. It is interesting to note that the new colour structure $d^{a b c} d_{a b c}$ entering the valence splitting function $P_{\mathrm{v}}$ at three loops contributes only non-leading terms $(1-x)^{k \geq 1} \ln (1-x)$, in striking contrast to its dominance in the small- $x$ limit [6]. As indicated the first three terms in Eq. (3.2) are the same for all three splitting functions $P_{a}$ - with the non-vanishing $(l>0)$ coefficients $\widetilde{C}_{l+1}$ being combinations of lower-order cusp anomalous dimensions $A_{n \leq l}$ - and their functional form is independent on the perturbative order $l$. This independence is established to all orders in Ref. [5] for the first two terms, and strongly suggested for the third term by the conjecture of Ref. [8] and its third-order verification in Refs. [9, 10], see also Ref. [49].

Eq. (3.2) also holds for the corresponding timelike splitting functions [9, 48] governing the evolution of the non-singlet fragmentation densities, with the same large- $x$ coefficients except for a sign change of $\widetilde{C}$ relative to the spacelike case [8]. Hence it appears that none of the non-singlet splitting functions exhibits any large- $x$ double logarithms at any order of $(1-x)$.

The known coefficient functions for the deep-inelastic structure functions (2.1) and the fragmentation functions (2.10) - with the obvious exception of $\mathcal{F}_{L}$ - receive the same highest double logarithmic contributions,

$$
c_{a, l}(x)=\frac{1}{(l-1) !}\left(2 C_{F}\right)^{l} p_{q q}(x) \ln ^{2 l-1}(1-x)+o\left((1-x)^{k \geq-1} \ln ^{2 l-2}(1-x)\right)
$$

with

$$
p_{q q}(x)=\frac{2}{(1-x)_{+}}-2+(1-x)
$$

and $C_{F}=\left(2 N_{c}\right)^{-1}\left(N_{c}^{2}-1\right)=4 / 3$ in QCD. Eq. (3.3) conforms to the general observation, going back to Ref. [50], that the coefficient of the highest $(1-x)^{0}$ logarithm is the negative of that of the highest +-distribution. Actually, this pattern also applies to the $C_{F}^{l-1}\left\{C_{A}, n_{f}\right\} \ln ^{2 l-2}(1-x)$ and $C_{F}^{l-2}\left\{C_{A}^{2}, C_{A} n_{f}, n_{f}^{2}\right\} \ln ^{2 l-3}(1-x)$ terms to $l=3$, see Refs. [27,28]. Analogous results, e.g.,

$$
c_{\mathrm{DY}, l}(x)=\frac{1}{(l-1) !}\left(8 C_{F}\right)^{l} p_{q q}(x) \ln ^{2 l-1}(1-x)+o\left((1-x)^{k \geq-1} \ln ^{2 l-2}(1-x)\right)
$$

hold for the Drell-Yan cross section (2.11). 
For the convenience of the reader, we now proceed to provide the full $\ln ^{n}(1-x)$ contributions to all known coefficient functions as far as they are relevant for our main predictions in later sections. The coefficient functions (2.2) for $\mathcal{F}_{1}$ read

$$
\begin{aligned}
& c_{1,1}(x)=\ln (1-x) 2 C_{F} p_{q q}(x) \\
& +C_{F}\left\{p_{q q}(x)\left(-3 / 2-2 \mathrm{H}_{0}\right)-\delta(1-x)\left(9+4 \zeta_{2}\right)+3+3 / 2(1-x)\right\}, \\
& c_{1,2}(x)=\left(\ln ^{3}(1-x) 4 C_{F}^{2}-\ln ^{2}(1-x) C_{F} \beta_{0}\right) p_{q q}(x) \\
& +\ln ^{2}(1-x)\left[C_{F}^{2}\left\{p_{q q}(x)\left(-9-14 \mathrm{H}_{0}\right)+6+4 \mathrm{H}_{0}-(1-x)\left(1+2 \mathrm{H}_{0}\right)\right\}\right] \\
& +\ln (1-x)\left[C _ { F } ^ { 2 } \left\{p_{q q}(x)\left(-27 / 2-4 \widetilde{\mathrm{H}}_{1,0}+24 \mathrm{H}_{0,0}+12 \mathrm{H}_{0}-16 \zeta_{2}\right)+8 \widetilde{\mathrm{H}}_{1,0}\right.\right. \\
& \left.-11-16 \mathrm{H}_{0,0}-32 \mathrm{H}_{0}+(1-x)\left(-53 / 2-4 \widetilde{\mathrm{H}}_{1,0}+8 \mathrm{H}_{0,0}+12 \mathrm{H}_{0}+8 \zeta_{2}\right)\right\} \\
& +C_{F} \beta_{0}\left\{p_{q q}(x)\left(29 / 6+4 \mathrm{H}_{0}\right)-3+5 / 2(1-x)\right\} \\
& +C_{F} C_{A}\left\{p_{q q}(x)\left(8 / 3+4 \widetilde{\mathrm{H}}_{1,0}+4 \mathrm{H}_{0,0}-4 \zeta_{2}\right)+2+(1-x)\left(14-4 \zeta_{2}\right)\right\} \\
& \left.+C_{F}\left(C_{A}-2 C_{F}\right) p_{q q}(-x)\left(8 \widetilde{\mathrm{H}}_{-1,0}-4 \mathrm{H}_{0,0}\right)\right] \\
& +o\left(\ln ^{0}(1-x)\right) \\
& c_{1,3}(x)=\left(\ln ^{5}(1-x) 4 C_{F}^{3}-\ln ^{4}(1-x) 10 / 3 C_{F}^{2} \beta_{0}+\ln ^{3}(1-x) 2 / 3 C_{F} \beta_{0}^{2}\right) p_{q q}(x) \\
& +\ln ^{4}(1-x)\left[C_{F}^{3}\left\{p_{q q}(x)\left(-15-24 \mathrm{H}_{0}\right)+6+8 \mathrm{H}_{0}-(1-x)\left(5+4 \mathrm{H}_{0}\right)\right\}\right] \\
& +\ln ^{3}(1-x)\left[C _ { F } ^ { 3 } \left\{p_{q q}(x)\left(-18-8 \widetilde{\mathrm{H}}_{1,0}+296 / 3 \mathrm{H}_{0,0}+54 \mathrm{H}_{0}-48 \zeta_{2}\right)+32 \widetilde{\mathrm{H}}_{1,0}\right.\right. \\
& \left.-22-64 \mathrm{H}_{0,0}-84 \mathrm{H}_{0}-(1-x)\left(33+16 \widetilde{\mathrm{H}}_{1,0}-32 \mathrm{H}_{0,0}-54 \mathrm{H}_{0}-16 \zeta_{2}\right)\right\} \\
& +C_{F}^{2} \beta_{0}\left\{p_{q q}(x)\left(70 / 3+164 / 9 \mathrm{H}_{0}\right)-8-4 \mathrm{H}_{0}+(1-x)\left(8+2 \mathrm{H}_{0}\right)\right\} \\
& +C_{F}^{2} C_{A}\left\{p_{q q}(x)\left(32 / 3+8 \widetilde{\mathrm{H}}_{1,0}+8 \mathrm{H}_{0,0}-16 \zeta_{2}\right)+4+(1-x)\left(28-8 \zeta_{2}\right)\right\} \\
& \left.+C_{F}^{2}\left(C_{A}-2 C_{F}\right) p_{q q}(-x)\left(16 \widetilde{\mathrm{H}}_{-1,0}-8 \mathrm{H}_{0,0}\right)\right] \\
& +O\left(\ln ^{2}(1-x)\right) \text {. }
\end{aligned}
$$

Here and below we suppress the argument $x$ of the harmonic polylogarithms [44] for brevity. Furthermore we use a slightly non-standard set of basis functions, i.e., Eqs. (3.7) and (3.8) include

$$
\begin{aligned}
\widetilde{\mathrm{H}}_{1,0}(x) & =\mathrm{H}_{1,0}(x)+\zeta_{2}=-\ln x \ln (1-x)-\mathrm{Li}_{2}(x)+\zeta_{2}, \\
\widetilde{\mathrm{H}}_{-1,0}(x) & =\mathrm{H}_{-1,0}(x)+\zeta_{2} / 2=\ln x \ln (1+x)+\mathrm{Li}_{2}(-x)+\zeta_{2} / 2
\end{aligned}
$$

besides $\mathrm{H}_{0}(x)=\ln x$ and $\mathrm{H}_{0,0}(x)=1 / 2 \ln ^{2} x$. All (modified) H-functions employed in our equations have a Taylor expansion at $x=1$, starting at order $(1-x)$ or higher, with rational coefficients. Thus also all terms with the Riemann $\zeta$-function can be read off directly from our expansions. 
The corresponding results for the structure function $\mathcal{F}_{2}$ in 2.1 are given by

$$
\begin{aligned}
& c_{2,1}(x)=c_{1,1}(x)+4 x C_{F} \\
& c_{2,2}(x)=c_{1,2}(x)+\ln ^{2}(1-x) 8 x C_{F}^{2} \\
& +\ln (1-x)\left[C_{F}^{2}\left\{8-x\left(4+24 \mathrm{H}_{0}\right)\right\}-4 x C_{F} \beta_{0}-C_{F}\left(C_{A}-2 C_{F}\right) 16 x\left(1-\zeta_{2}\right)\right] \\
& +C_{F}^{2}\left\{12-8 \mathrm{H}_{0}-\left(130 / 3+8 \widetilde{\mathrm{H}}_{1,0}-16 \mathrm{H}_{0,0}+16 \zeta_{2}\right) x\right\} \\
& -C_{F} \beta_{0}\left\{4-\left(50 / 3+8 \mathrm{H}_{0}\right) x\right\} \\
& +C_{F}\left(C_{A}-2 C_{F}\right)\left\{-32 /\left(5 x^{2}\right)\left(\widetilde{\mathrm{H}}_{-1,0}-\zeta_{2} / 2\right)-32 /(5 x)\left(1-\mathrm{H}_{0}\right)\right. \\
& +8 / 5-32 \widetilde{\mathrm{H}}_{-1,0}-16 / 5 \mathrm{H}_{0}+16 \zeta_{2}+x\left(236 / 15+16\left[2 \widetilde{\mathrm{H}}_{-1,-1,0}\right.\right. \\
& \left.\left.-\widetilde{\mathrm{H}}_{-1,0,0}+\widetilde{\mathrm{H}}_{1,0,0}-\widetilde{\mathrm{H}}_{-1,0}+\mathrm{H}_{0,0}\right]+104 / 5 \mathrm{H}_{0}-8 \zeta_{2}-24 \zeta_{3}\right) \\
& \left.-48 / 5 x^{2}\left(1+\mathrm{H}_{0}\right)+48 / 5 x^{3}\left(\widetilde{\mathrm{H}}_{-1,0}-\mathrm{H}_{0,0}+\zeta_{2} / 2\right)\right\} \text {, } \\
& c_{2,3}(x)=c_{1,3}(x)+\ln ^{4}(1-x) 8 x C_{F}^{3} \\
& +\ln ^{3}(1-x)\left[C_{F}^{3}\left\{16-x\left(8+48 \mathrm{H}_{0}\right)\right\}-32 / 3 x C_{F}^{2} \beta_{0}-C_{F}^{2}\left(C_{A}-2 C_{F}\right) 32 x\left(1-\zeta_{2}\right)\right] \\
& +\ln ^{2}(1-x)\left[C_{F}^{3}\left\{16-48 \mathrm{H}_{0}-\left(166 / 3+32 \widetilde{\mathrm{H}}_{1,0}-160 \mathrm{H}_{0,0}-24 \mathrm{H}_{0}+96 \zeta_{2}\right) x\right\}\right. \\
& -C_{F}^{2} \beta_{0}\left\{20-\left(158 / 3+52 \mathrm{H}_{0}\right) x\right\}+C_{F} \beta_{0}^{2} 4 x \\
& +C_{F}^{2}\left(C_{A}-2 C_{F}\right)\left\{-64 /\left(5 x^{2}\right)\left(\widetilde{\mathrm{H}}_{-1,0}-\zeta_{2} / 2\right)-64 /(5 x)\left(1-\mathrm{H}_{0}\right)\right. \\
& -144 / 5-64 \widetilde{\mathrm{H}}_{-1,0}-32 / 5 \mathrm{H}_{0}+64 \zeta_{2}+x\left(872 / 15+32\left[2 \widetilde{\mathrm{H}}_{-1,-1,0}\right.\right. \\
& \left.\left.-\widetilde{\mathrm{H}}_{-1,0,0}+\widetilde{\mathrm{H}}_{1,0,0}-\widetilde{\mathrm{H}}_{-1,0}+\mathrm{H}_{0,0}\right]+688 / 5 \mathrm{H}_{0}-48 \zeta_{2}-96 \zeta_{2} \mathrm{H}_{0}-48 \zeta_{3}\right) \\
& \left.-96 / 5 x^{2}\left(1+\mathrm{H}_{0}\right)+96 / 5 x^{3}\left(\widetilde{\mathrm{H}}_{-1,0}-\mathrm{H}_{0,0}+\zeta_{2} / 2\right)\right\} \\
& \left.+C_{F}\left(C_{A}-2 C_{F}\right) \beta_{0} 24 x\left(1-\zeta_{2}\right)+C_{F}\left(C_{A}-2 C_{F}\right)^{2} 32 x\left(\zeta_{2}-\zeta_{3}\right)\right] \\
& +O(\ln (1-x)) \text {. }
\end{aligned}
$$

The differences $c_{2, l}-c_{1, l}$ are, of course, the coefficient functions for the longitudinal structure function in DIS. Hence we have included one more order in $\ln (1-x)$ in Eqs. (3.10) and (3.11). This order additionally includes a combination of three weight-three harmonic polylogarithms,

$$
\begin{aligned}
\widetilde{\mathrm{H}}_{-1,-1,0}(x) & =\mathrm{H}_{-1,-1,0}(x)+\mathrm{H}_{-1}(x) \zeta_{2} / 2-\zeta_{3} / 8 \\
\widetilde{\mathrm{H}}_{-1,0,0}(x) & =\mathrm{H}_{-1,0,0}(x)-3 \zeta_{3} / 4 \\
\widetilde{\mathrm{H}}_{1,0,0}(x) & =\mathrm{H}_{1,0,0}(x)-\zeta_{3}
\end{aligned}
$$

besides the unmodified $\mathrm{H}_{0,0,0}(x)=1 / 6 \ln ^{3} x$. The reader is referred to Ref. [24] for expressions of these functions in terms of the standard polylogarithms $\mathrm{Li}_{2}(x)$ and $\mathrm{Li}_{3}(x)$. 
To the same accuracy as Eqs. (3.6) - (3.8) for $\mathcal{F}_{1}$, the coefficient functions for $\mathcal{F}_{3}$ can be written as

$$
\begin{aligned}
c_{3,1}(x)= & c_{1,1}(x)-2 C_{F}(1-x) \\
c_{3,2}(x)= & c_{1,2}(x)-\ln ^{2}(1-x) 4 C_{F}^{2}(1-x) \\
+ & \ln (1-x)\left[C_{F}^{2}\left\{-32 \mathrm{H}_{0}+(1-x)\left(18+28 \mathrm{H}_{0}-16 \zeta_{2}\right)\right\}\right. \\
& \quad+2 C_{F} \beta_{0}(1-x)+C_{F} C_{A}\left\{16 \mathrm{H}_{0}-8(1-x)\left(1+\mathrm{H}_{0}-\zeta_{2}\right)\right\} \\
& \left.\quad-C_{F}\left(C_{A}-2 C_{F}\right) 8 p_{q q}(-x)\left(2 \widetilde{\mathrm{H}}_{-1,0}-\mathrm{H}_{0,0}\right)\right] \\
+ & o\left(\ln ^{0}(1-x)\right), \\
c_{3,3}(x)= & c_{1,3}(x)-\ln ^{4}(1-x) 4 C_{F}^{3}(1-x) \\
+ & \ln ^{3}(1-x)\left[C_{F}^{3}\left\{-64 \mathrm{H}_{0}+(1-x)\left(36+56 \mathrm{H}_{0}-32 \zeta_{2}\right)\right\}\right. \\
& +16 / 3 C_{F}^{2} \beta_{0}(1-x)+C_{F}^{2} C_{A}\left\{32 \mathrm{H}_{0}-16(1-x)\left(1+\mathrm{H}_{0}-\zeta_{2}\right)\right\} \\
& \left.\quad-C_{F}^{2}\left(C_{A}-2 C_{F}\right) 16 p_{q q}(-x)\left(2 \widetilde{\mathrm{H}}_{-1,0}-\mathrm{H}_{0,0}\right)\right] \\
+ & O\left(\ln ^{2}(1-x)\right) .
\end{aligned}
$$

As mentioned below Eq. (3.8), the H-functions in our expansions start at order $(1-x)$ or higher at large $x$. Hence one can directly read off from Eqs. (3.12) - (3.14) that the coefficient functions for $\mathcal{F}_{1}$ and $\mathcal{F}_{3}$ differ for $x \rightarrow 1$ only in terms of order $(1-x)$. This fact was already noted in Ref. [28].

The third-order coefficient functions (3.8), (3.11) and (3.14) receive contributions from new flavour classes involving the higher group invariant $d^{a b c} d_{a b c}$ [26-28]. The highest $d^{a b c} d_{a b c}$ terms behave as $(1-x) \ln (1-x)$ for $\mathcal{F}_{1}$ and $\mathcal{F}_{2}$, and as $(1-x) \ln ^{2}(1-x)$ for $\mathcal{F}_{3}$. Their leading contributions for the longitudinal structure function is of order $(1-x)^{2} \ln (1-x)$. These terms will not be relevant on the level of our present analysis. The same holds for the new three-loop functions $g_{i}(x)$ which also show only a single-logarithmic behaviour for $x \rightarrow 1$ [27,28].

The coefficient functions for the transverse fragmentation function $\mathcal{F}_{T}$ are related to those for $\mathcal{F}_{1}$ in DIS by suitably defined analytic continuations. Hence we also present their $\ln (1-x)$ expansions relative to the results for $c_{1, l}(x)$ in Eqs. (3.6) - (3.8):

$$
\begin{aligned}
c_{T, 1}(x)= & c_{1,1}(x)+C_{F}\left\{12 \zeta_{2} \delta(1-x)+6 p_{q q}(x) \mathrm{H}_{0}-6+3(1-x)\right\} \\
c_{T, 2}(x)= & c_{1,2}(x)+\ln ^{2}(1-x) C_{F}^{2}\left\{24 p_{q q}(x) \mathrm{H}_{0}-12+6(1-x)\right\} \\
+ & \ln (1-x)\left[C _ { F } ^ { 2 } \left\{p_{q q}(x)\left(8 \widetilde{\mathrm{H}}_{1,0}-28 \mathrm{H}_{0,0}-18 \mathrm{H}_{0}+24 \zeta_{2}\right)\right.\right. \\
& \left.\quad+22+40 \mathrm{H}_{0,0}+12 \mathrm{H}_{0}-(1-x)\left(11+20 \mathrm{H}_{0,0}+10 \mathrm{H}_{0}\right)\right\} \\
& +C_{F} \beta_{0}\left\{-6 p_{q q}(x) \mathrm{H}_{0}+6-3(1-x)\right\} \\
& \left.+C_{F} C_{A}\left\{p_{q q}(x)\left(-8 \widetilde{\mathrm{H}}_{1,0}-4 \mathrm{H}_{0,0}\right)-4+2(1-x)\right\}\right] \\
+ & o\left(\ln ^{0}(1-x)\right)
\end{aligned}
$$




$$
\begin{aligned}
c_{T, 3}(x)= & c_{1,3}(x)+\ln ^{4}(1-x) C_{F}^{3}\left\{36 p_{q q}(x) \mathrm{H}_{0}-12+6(1-x)\right\} \\
+ & \ln ^{3}(1-x)\left[C _ { F } ^ { 3 } \left\{p_{q q}(x)\left(16 \widetilde{\mathrm{H}}_{1,0}-104 \mathrm{H}_{0,0}-72 \mathrm{H}_{0}+48 \zeta_{2}\right)\right.\right. \\
& \left.\quad+44+128 \mathrm{H}_{0,0}+24 \mathrm{H}_{0}-(1-x)\left(22+64 \mathrm{H}_{0,0}+44 \mathrm{H}_{0}\right)\right\} \\
& +C_{F}^{2} \beta_{0}\left\{-232 / 9 p_{q q}(x) \mathrm{H}_{0}+16-8(1-x)\right\} \\
& \left.+C_{F}^{2} C_{A}\left\{p_{q q}(x)\left(-16 \widetilde{\mathrm{H}}_{1,0}-8 \mathrm{H}_{0,0}\right)-8+4(1-x)\right\}\right] \\
+ & o\left(\ln ^{2}(1-x)\right) .
\end{aligned}
$$

As for the spacelike case of Eqs. (3.9) - (3.11), also the coefficient functions for the timelike longitudinal structure function $\mathcal{F}_{L}$ in Eq. (2.10) will be needed to one more order in $\ln (1-x)$ below. Their corresponding expansions are given by

$$
\begin{aligned}
& c_{L, 1}(x)=2 C_{F}, \\
& c_{L, 2}(x)=\ln ^{2}(1-x) 4 C_{F}^{2} \\
& +\ln (1-x)\left[C_{F}^{2}\left\{-2+4 \mathrm{H}_{0}+4 x\right\}-2 C_{F} \beta_{0}-8 C_{F}\left(C_{A}-2 C_{F}\right)\left(1-\zeta_{2}\right)\right] \\
& +C_{F}^{2}\left\{-41 / 3-12 \widetilde{\mathrm{H}}_{1,0}-12 \mathrm{H}_{0,0}+2 \mathrm{H}_{0}+16 \zeta_{2}-x\left(2-8 \mathrm{H}_{0}\right)\right\} \\
& +C_{F} \beta_{0}\left\{25 / 3-2 \mathrm{H}_{0}-2 x\right\} \\
& +C_{F}\left(C_{A}-2 C_{F}\right)\left\{-24 /\left(5 x^{2}\right)\left(\widetilde{\mathrm{H}}_{-1,0}-\zeta_{2} / 2\right)-24 /(5 x)\left(1-\mathrm{H}_{0}\right)+118 / 15\right. \\
& -8\left[2 \widetilde{\mathrm{H}}_{-1,-1,0}-\widetilde{\mathrm{H}}_{-1,0,0}-2 \widetilde{\mathrm{H}}_{0,-1,0}-\widetilde{\mathrm{H}}_{1,0,0}-\widetilde{\mathrm{H}}_{-1,0}\right]-12 / 5 \mathrm{H}_{0} \\
& -4 \zeta_{2}-8 \zeta_{2} \mathrm{H}_{0}-12 \zeta_{3}+x\left(4 / 5+16 \widetilde{\mathrm{H}}_{-1,0}-16 \mathrm{H}_{0,0}+8 / 5 \mathrm{H}_{0}+8 \zeta_{2}\right) \\
& \left.-16 / 5 x^{2}\left(1+\mathrm{H}_{0}\right)+16 / 5 x^{3}\left(\widetilde{\mathrm{H}}_{-1,0}-\mathrm{H}_{0,0}+\zeta_{2} / 2\right)\right\} \text {, } \\
& c_{L, 3}(x)=\ln ^{4}(1-x) 4 C_{F}^{3} \\
& +\ln ^{3}(1-x)\left[C_{F}^{3}\left\{-4+8 \mathrm{H}_{0}+8 x\right\}-16 / 3 C_{F}^{2} \beta_{0}-16 C_{F}^{2}\left(C_{A}-2 C_{F}\right)\left(1-\zeta_{2}\right)\right] \\
& +\ln ^{2}(1-x)\left[C_{F}^{3}\left\{-35 / 3-16 \widetilde{\mathrm{H}}_{1,0}-16 \mathrm{H}_{0,0}-x\left(8-16 \mathrm{H}_{0}\right)\right\}\right. \\
& +C_{F}^{2} \beta_{0}\left\{79 / 3-10 \mathrm{H}_{0}-10 x\right\} \\
& +C_{F}^{2}\left(C_{A}-2 C_{F}\right)\left\{-48 /\left(5 x^{2}\right)\left(\widetilde{\mathrm{H}}_{-1,0}-\zeta_{2} / 2\right)-48 /(5 x)\left(1-\mathrm{H}_{0}\right)+436 / 15\right. \\
& -16\left[2 \widetilde{\mathrm{H}}_{-1,-1,0}-\widetilde{\mathrm{H}}_{-1,0,0}-2 \widetilde{\mathrm{H}}_{0,-1,0}-\widetilde{\mathrm{H}}_{1,0,0}-\widetilde{\mathrm{H}}_{-1,0}\right]-104 / 5 \mathrm{H}_{0} \\
& -24 \zeta_{2}-24 \zeta_{3}+x\left(-72 / 5+32 \widetilde{\mathrm{H}}_{-1,0}-32 \mathrm{H}_{0,0}+16 / 5 \mathrm{H}_{0}+32 \zeta_{2}\right) \\
& \left.-32 / 5 x^{2}\left(1+\mathrm{H}_{0}\right)+32 / 5 x^{3}\left(\widetilde{\mathrm{H}}_{-1,0}-\mathrm{H}_{0,0}+\zeta_{2} / 2\right)\right\} \\
& \left.+2 C_{F} \beta_{0}^{2}+C_{F}\left(C_{A}-2 C_{F}\right) \beta_{0} 12\left(1-\zeta_{2}\right)+C_{F}\left(C_{A}-2 C_{F}\right)^{2} 16\left(\zeta_{2}-\zeta_{3}\right)\right] \\
& +O(\ln (1-x)) \text {. }
\end{aligned}
$$


The last two equations include one more modified harmonic polylogarithm,

$$
\widetilde{\mathrm{H}}_{0,-1,0}(x)=\mathrm{H}_{0,-1,0}(x)+\mathrm{H}_{0}(x) \zeta_{2} / 2+3 \zeta_{3} / 2 \text {. }
$$

The final observable in Eq. (2.10), the asymmetric fragmentation function $\mathcal{F}_{A}$, is analogous to the structure function $\mathcal{F}_{3}$ in DIS. The $\alpha_{\mathrm{s}}$-expansion (2.2) of its coefficient function reads

$$
\begin{aligned}
c_{A, 1}(x)= & c_{T, 1}(x)-2 C_{F}(1-x), \\
c_{A, 2}(x)= & c_{T, 2}(x)-\ln ^{2}(1-x) 4 C_{F}^{2}(1-x) \\
+ & \ln (1-x)\left[C_{F}^{2}\left\{-32 \mathrm{H}_{0}+(1-x)\left(18+12 \mathrm{H}_{0}-16 \zeta_{2}\right)\right\}\right. \\
& \quad+2 C_{F} \beta_{0}(1-x)+C_{F} C_{A}\left\{16 \mathrm{H}_{0}-8(1-x)\left(1+\mathrm{H}_{0}-\zeta_{2}\right)\right\} \\
& \left.\quad-C_{F}\left(C_{A}-2 C_{F}\right) 8 p_{q q}(-x)\left(2 \widetilde{\mathrm{H}}_{-1,0}-\mathrm{H}_{0,0}\right)\right] \\
+ & o\left(\ln ^{0}(1-x)\right), \\
c_{A, 3}(x)= & c_{T, 3}(x)-\ln ^{4}(1-x) 4 C_{F}^{3}(1-x) \\
+ & \ln ^{3}(1-x)\left[C_{F}^{3}\left\{-64 \mathrm{H}_{0}+(1-x)\left(36+24 \mathrm{H}_{0}-32 \zeta_{2}\right)\right\}\right. \\
& +16 / 3 C_{F}^{2} \beta_{0}(1-x)+C_{F}^{2} C_{A}\left\{32 \mathrm{H}_{0}-16(1-x)\left(1+\mathrm{H}_{0}-\zeta_{2}\right)\right\} \\
& \left.\quad-C_{F}^{2}\left(C_{A}-2 C_{F}\right) 16 p_{q q}(-x)\left(2 \widetilde{\mathrm{H}}_{-1,0}-\mathrm{H}_{0,0}\right)\right] \\
+ & o\left(\ln ^{2}(1-x)\right) .
\end{aligned}
$$

The relations (3.17), (3.20) and (3.23) for the third-order timelike coefficient functions have not been presented before. These results have been obtained by extending the analytic continuations of Ref. [9] to terms of order $\alpha_{s}^{3} \varepsilon^{0}$ in dimensional regularization. At the end of this section we will present sufficient evidence that, for these $\ln (1-x)$ contributions, this analytic continuation does not suffer from the $\pi^{2}$-problem mentioned below Eq. (2.10).

Finally the known coefficient functions for the Drell-Yan cross section (2.11) are given by

$$
\begin{aligned}
c_{\mathrm{DY}, 1}(x)= & \ln (1-x) 8 C_{F} p_{q q}(x)+C_{F}\left\{-4 p_{q q}(x) \mathrm{H}_{0}-\delta(1-x)\left(16-8 \zeta_{2}\right)\right\} \\
c_{\mathrm{DY}, 2}(x)= & \left(\ln ^{3}(1-x) 64 C_{F}^{2}-8 \ln ^{2}(1-x) C_{F} \beta_{0}\right) p_{q q}(x) \\
+ & \ln ^{2}(1-x)\left[C_{F}^{2}\left\{-124 p_{q q}(x) \mathrm{H}_{0}+64 \mathrm{H}_{0}-(1-x)\left(64+32 \mathrm{H}_{0}\right)\right\}\right] \\
+ & \ln (1-x)\left[C _ { F } ^ { 2 } \left\{p_{q q}(x)\left(-128-8 \widetilde{\mathrm{H}}_{1,0}+112 \mathrm{H}_{0,0}-24 \mathrm{H}_{0}-64 \zeta_{2}\right)-4\right.\right. \\
& \left.\quad+96\left[\widetilde{\mathrm{H}}_{1,0}-\mathrm{H}_{0,0}\right]-160 \mathrm{H}_{0}+(1-x)\left(4-48 \widetilde{\mathrm{H}}_{1,0}+48 \mathrm{H}_{0,0}+168 \mathrm{H}_{0}\right)\right\} \\
& +C_{F} \beta_{0}\left\{p_{q q}(x)\left(40 / 3+16 \mathrm{H}_{0}\right)+16(1-x)\right\} \\
& +C_{F} C_{A}\left\{p_{q q}(x)\left(32 / 3+8 \widetilde{\mathrm{H}}_{1,0}+16 \mathrm{H}_{0,0}-16 \zeta_{2}\right)+4+16 \widetilde{\mathrm{H}}_{1,0}\right. \\
& \left.\left.\quad+32 \mathrm{H}_{0}+(1-x)\left(44-8 \widetilde{\mathrm{H}}_{1,0}-16 \mathrm{H}_{0}\right)\right\}\right] \\
+ & O\left(\ln ^{0}(1-x)\right) .
\end{aligned}
$$


At the third order only the +-distributions contributions $\left[(1-x)^{-1} \ln ^{n}(1-x)\right]_{+}, n=0, \ldots, 5$ are known so far, see Ref. [13].

Eqs. (3.6) - 3.23) can be employed to derive the $\ln (1-x)$ expansion of the physical evolution kernels (2.8) for the deep-inelastic structure functions (2.1) and the $e^{+} e^{-}$fragmentation functions $\mathcal{F}_{T}, \mathcal{F}_{T}+\mathcal{F}_{L}$ and $\mathcal{F}_{A}$ of Eq. (2.10). Recalling that $K_{a, n}$ denotes the $\mathrm{N}^{n} \mathrm{LO}$ kernel for $\mathcal{F}_{a}$, one finds

$$
\begin{aligned}
K_{a, 0}(x) & =2 C_{F} p_{q q}(x)+3 C_{F} \delta(1-x) \\
K_{a, 1}(x) & =\ln (1-x) p_{q q}(x)\left[-2 C_{F} \beta_{0} \mp 8 C_{F}^{2} \mathrm{H}_{0}\right]+o\left(\ln ^{0}(1-x)\right) \\
K_{a, 2}(x) & =\ln ^{2}(1-x) p_{q q}(x)\left[2 C_{F} \beta_{0}^{2} \pm 12 C_{F}^{2} \beta_{0} \mathrm{H}_{0}+32 C_{F}^{3} \mathrm{H}_{0,0}\right]+o(\ln (1-x)), \\
K_{a, 3}(x) & =\ln ^{3}(1-x) p_{q q}(x)\left[-2 C_{F} \beta_{0}^{3} \mp 44 / 3 C_{F}^{2} \beta_{0}^{2} \mathrm{H}_{0}-64 C_{F}^{3} \beta_{0} \mathrm{H}_{0,0}+\xi_{P_{3}} C_{F}^{4} \mathrm{H}_{0,0,0}\right] \\
& +o\left(\ln ^{2}(1-x)\right) .
\end{aligned}
$$

From the NLO result $K_{a, 1}$ we have only written down the leading $\ln (1-x)$ terms. These contributions are the same for all six structure functions up to a sign change of the $\mathrm{H}_{0}$ terms between the DIS quantities (upper sign) and the fragmentation functions (lower sign). The non- $\beta_{0}$ terms in Eqs. (3.26) are the contributions of the $\overline{\mathrm{MS}}$ splitting functions (3.2), consequently the fourth-order coefficient $\xi_{P_{3}}$ is unknown at this point, but irrelevant for our further considerations.

The corresponding results for the Drell-Yan cross section (2.11) are given by

$$
\begin{aligned}
K_{\mathrm{DY}, 0}(x) & =4 C_{F} p_{q q}(x)+6 C_{F} \delta(1-x) \\
K_{\mathrm{DY}, 1}(x) & =\ln (1-x) p_{q q}(x)\left[-8 C_{F} \beta_{0}-16 C_{F}^{2} \mathrm{H}_{0}\right]+o\left(\ln ^{0}(1-x)\right), \\
K_{\mathrm{DY}, 2}(x) & =\ln ^{2}(1-x) p_{q q}(x)\left[16 C_{F} \beta_{0}^{2}+56 C_{F}^{2} \beta_{0} \mathrm{H}_{0}+64 C_{F}^{3} \mathrm{H}_{0,0}\right]+o(\ln (1-x)) .
\end{aligned}
$$

Eqs. (3.26) and (3.27) represent our crucial observation: the physical kernels for all seven non-singlet observables display an only single-logarithmic large- $x$ enhancement, at all powers of $(1-x)$, to all orders in $\alpha_{\mathrm{s}}$ for which the corresponding coefficient functions are known. We consider it extremely unlikely that this pattern is accidental, and hence conjecture a single-logarithmic behaviour of these physical at (all) higher orders in $\alpha_{s}$, with the leading contribution showing the same independence on the specific structure function as in Eqs. (3.26). In support of this conjecture we note that for the +-distribution parts of $K_{a, n}$ (including the Drell-Yan case), recall Eq. (3.4), this single-logarithmic enhancement is established by the soft-gluon exponentiation as explained in the next section. Furthermore the all-order leading- $n_{f}$ results of Ref. [36] prove the all-order generalization of Eqs. (3.26) for the $C_{F} \beta_{0}^{n}$ contributions to the DIS kernels $K_{1, n}$ and $K_{2, n}$.

The single-logarithmic enhancement of the physical kernels directly leads to predictions for the highest $\ln (1-x)$ terms of the higher-order coefficient functions. Considering, for example, the third ( $\left.\mathrm{N}^{3} \mathrm{LO}\right)$ line of Eqs. (2.9), one notes that the convolutions of $c_{a, 1}(x)$ and $c_{a, 2}(x)$ lead to terms up to $\ln ^{5}(1-x)$. The vanishing of terms higher than $\ln ^{3}(1-x)$ thus fixes the $\ln ^{4}(1-x)$ and $\ln ^{5}(1-x)$ terms of $c_{a, 3}(x)$. In fact, exactly this reasoning, together with the absence of any $\zeta_{2}$ terms in Eqs. (3.26), provides the additional confirmation of the correctness of Eqs. (3.17), (3.20) and (3.23) mentioned below the latter equation. 


\section{Soft-gluon exponentiation of the leading contributions}

The leading (+-distribution) large- $x$ terms of the above coefficient functions can be expressed to all orders in $\alpha_{\mathrm{s}}$ in terms of the soft-gluon exponentiation [11]. Switching to the Mellin moments defined in Eq. (2.7), these contributions to Eq. (2.2) can be written as

$$
C(N)=g_{0}\left(a_{\mathrm{s}}\right) \exp \left\{\ln N g_{1}(\lambda)+g_{2}(\lambda)+a_{\mathrm{s}} g_{3}(\lambda)+o\left(a_{\mathrm{s}}^{2} f(\lambda)\right)\right\}
$$

up to terms which vanish for $N \rightarrow \infty$. Here we have used the standard abbreviation

$$
\lambda=a_{\mathrm{s}} \beta_{0} L \equiv \frac{\alpha_{\mathrm{s}}}{4 \pi} \beta_{0} L \quad \text { with } \quad L \equiv \ln N,
$$

and we have again put $\mu_{\mathrm{r}}=\mu_{\mathrm{f}}=Q$. By virtue of the first line of Eq. (2.8) - the logarithmic derivative in $N$-space - Eq. (4.1) leads to the following expression for the resummed kernels up to next-to-next-to-leading logarithmic (NNLL) accuracy [21]:

$$
\begin{aligned}
K_{a}(N)= & -\eta_{a}\left(A_{1} a_{\mathrm{s}}+A_{2} a_{\mathrm{s}}^{2}+A_{3} a_{\mathrm{s}}^{3}\right) \ln N-\left(1+\frac{\beta_{1}}{\beta_{0}} a_{\mathrm{s}}+\frac{\beta_{2}}{\beta_{0}} a_{\mathrm{s}}^{2}\right) \lambda^{2} \frac{d g_{a, 1}}{d \lambda} \\
& -\left(a_{\mathrm{s}} \beta_{0}+a_{\mathrm{s}}^{2} \beta_{1}\right) \lambda \frac{d g_{a, 2}}{d \lambda}-a_{\mathrm{s}}^{2} \beta_{0} \frac{d}{d \lambda}\left(\lambda g_{a, 3}(\lambda)\right)+o\left(a_{s}^{3}(f(\lambda))\right.
\end{aligned}
$$

with $\eta_{a}=2$ for $a=\mathrm{DY}$ and $\eta_{a}=1$ otherwise, cf. the last paragraph of Section 2 . Thus the leading logarithmic (LL), next-to-leading logarithmic (NLL) and NNLL large- $N$ contributions to the physical kernels are of the form $\left(a_{S} \ln N\right)^{n}, a_{s}\left(a_{S} \ln N\right)^{n}$ and $a_{S}^{2}\left(a_{S} \ln N\right)^{n}$, respectively. Recalling

$$
f(N)=\frac{(-1)^{n}}{n} \ln ^{n} N+O\left(\ln ^{n-2} N\right) \quad \text { for } \quad f(x)=\left[\frac{\ln ^{n-1}(1-x)}{1-x}\right]_{+},
$$

one notes that Eq. (4.3) implies that the single-logarithmic enhancement (3.26) and (3.27) holds to all order in $\alpha_{s}$ for the +-distribution contributions.

In the next two sections we will provide analogous all-order results for the subleading $N^{-1} \ln ^{n} N$ contributions to the coefficient functions. These results, however, will be restricted to a towerexpanded NLL accuracy, see Ref. [51]. The exponents analogous to Eq. (4.1) will be given relative to the LL and NLL functions entering the soft-gluon exponent. For the deep-inelastic structure functions $\mathcal{F}_{1,2,3}$ and the fragmentation functions $\mathcal{F}_{T+L, T, A}$ these functions read

$$
\begin{aligned}
g_{a, 1}\left(a_{\mathrm{s}} L\right)= & \frac{A_{1}}{\beta_{0} \lambda}[\lambda+(1-\lambda) \ln (1-\lambda)] \equiv \sum_{k=1} g_{1 k}\left(a_{\mathrm{s}} L\right)^{k} \\
= & \sum_{k=1}^{\infty} \frac{A_{1} \beta_{0}^{k-1}}{k(k+1)}\left(a_{\mathrm{s}} L\right)^{k} \\
g_{a, 2}\left(a_{\mathrm{s}} L\right)= & -\frac{\gamma_{\mathrm{e}} A_{1}-B_{1}}{\beta_{0}} \ln (1-\lambda)-\frac{A_{2}}{\beta_{0}^{2}}[\lambda+\ln (1-\lambda)] \\
& +\frac{A_{1} \beta_{1}}{\beta_{0}^{3}}\left[\lambda+\ln (1-\lambda)+\frac{1}{2} \ln ^{2}(1-\lambda)\right] \equiv \sum_{k=1} g_{2 k}\left(a_{\mathrm{s}} L\right)^{k} \\
= & \sum_{k=1}^{\infty}\left\{\frac{\gamma_{\mathrm{e}} A_{1}-B_{1}}{\beta_{0}}+\theta_{k 2}\left(\frac{A_{2}}{\beta_{0}^{2}}+\frac{A_{1} \beta_{1}}{\beta_{0}^{3}}\left[S_{1}(k-1)-1\right]\right)\right\} \frac{\beta_{0}^{k}}{k}\left(a_{\mathrm{s}} L\right)^{k}
\end{aligned}
$$


with $\theta_{k j}=1$ for $k \geq j$ and $\theta_{k j}=0$ else, and $S_{1}(k)=\sum_{j=1}^{k} 1 / j$ [51]. Here $A_{1}$ and $A_{2}$ are the oneand two-loop +-distribution coefficients in Eq. (3.2), given by [52]

$$
A_{1}=4 C_{F}, \quad A_{2}=8 C_{F} K=8 C_{F}\left[\left(\frac{67}{18}-\zeta_{2}\right) C_{A}-\frac{5}{9} n_{f}\right] .
$$

Note that, besides these expansion coefficients and those of the beta function (2.4), only one additional coefficient,

$$
B_{1}=-3 C_{F}
$$

enters the function $g_{a, 2}$ in Eq. (4.5) [11]. This pattern does persist at higher orders of the exponentiation $[22,25]$. Consequently the functions $g_{n>1}$ are completely fixed by the first term of their respective expansion in $\alpha_{s}$, if the cusp anomalous dimension and the beta function are known to a sufficient accuracy. To a large extent the predictive power of the soft-gluon exponentiation rests on this fact: the calculation of the $\mathrm{N}^{n} \mathrm{LO}$ coefficient function is sufficient to also determine the $\mathrm{N}^{n} \mathrm{LL}$ resummation function $g_{n+1}$, and thus two additional all-order towers of logarithms, see, e.g., Ref. [12]. As we will see below, however, this situation does not directly generalize to the non-leading large- $x /$ large- $N$ terms addressed in the next two sections.

The LL and NLL resummation exponents for the Drell-Yan cross section (2.11) are related to Eqs. (4.4) and (4.5) as follows:

$$
\begin{aligned}
& g_{\mathrm{DY}, 1}(\lambda)=2 g_{\mathrm{DIS}, 1}(2 \lambda), \\
& g_{\mathrm{DY}, 2}(\lambda)=g_{\mathrm{DIS}, 2}(2 \lambda) \text { with } \quad B_{1} \rightarrow 0 \text { and } \gamma_{\mathrm{e}} \rightarrow 2 \gamma_{\mathrm{e}} .
\end{aligned}
$$

Here, as above, $\gamma_{\mathrm{e}} \simeq 0.577216$ denotes the Euler-Mascheroni constant. The absence of any NLL resummation coefficient additional to $A_{1}$ and $\beta_{1}$ is a low-order 'accident', non-vanishing coefficients $D_{\mathrm{DY}, \mathrm{n}}$ occur at NNLL [53] and all higher orders.

For later convenience we finally recall the leading contributions $g_{31}$, defined as for $g_{1}$ and $g_{2}$ in Eqs. (4.4) and (4.5), to the NNLL resummation function in Eq. (4.1). The universal coefficient for DIS and $e^{+} e^{-}$annihilation, first extracted in Ref. [51] from the NNLO result of Refs. [23], reads

$$
\begin{aligned}
g_{a, 31} & =\left(\frac{3155}{54}-\frac{22}{3} \zeta_{2}-40 \zeta_{3}-8 \zeta_{2} \gamma_{\mathrm{e}}+\frac{22}{3} \gamma_{\mathrm{e}}^{2}+\frac{367}{9} \gamma_{\mathrm{e}}\right) C_{F} C_{A} \\
& +\left(\frac{3}{2}-12 \zeta_{2}+24 \zeta_{3}\right) C_{F}^{2}-\left(\frac{247}{27}-\frac{4}{3} \zeta_{2}+\frac{4}{3} \gamma_{\mathrm{e}}^{2}+\frac{58}{9} \gamma_{\mathrm{e}}\right) C_{F} n_{f}
\end{aligned}
$$

The corresponding result for the Drell-Yan case $[31,53]$ is given by

$$
\begin{aligned}
g_{\mathrm{DY}, 31} & =\left(\frac{1616}{27}-56 \zeta_{3}-32 \zeta_{2} \gamma_{\mathrm{e}}+\frac{176}{3} \gamma_{\mathrm{e}}^{2}+\frac{1072}{9} \gamma_{\mathrm{e}}\right) C_{F} C_{A} \\
& -\left(\frac{224}{27}+\frac{32}{3} \gamma_{\mathrm{e}}^{2}+\frac{160}{9} \gamma_{\mathrm{e}}\right) C_{F} n_{f} .
\end{aligned}
$$

Having collected all relevant fixed-order and soft-gluon resummation information, we can now turn to our new higher-order predictions. 


\section{Non-leading large- $N /$ large- $x$ terms in structure functions}

Keeping only the leading and subleading contributions, the large- $N$ behaviour of the coefficient functions (2.2) for the structure functions $\mathcal{F}_{a}$ in Eq. (2.1) can be written as

$$
c_{a, n}(N)=\sum_{k=0}^{2 n} c_{n k} L^{k}+\frac{1}{N} \sum_{k=0}^{2 n-1} d_{a, k}^{(n)} L^{k}+O\left(\frac{1}{N^{2}} L^{2 n-1}\right)
$$

with, as in the previous section, $L \equiv \ln N$. At the present accuracy the leading soft-gluon coefficients do not depend on the structure function, thus we have written $c_{n k}$ instead of $c_{a, k}^{(n)}$. $C_{1}$ and $C_{3}$ are identical at the level of Eq. (5.1) as discussed below Eq. (3.14) - recall that $N^{-2} \ln ^{a} N$ corresponds to $(1-x) \ln ^{a}(1-x)$. Note that the second sum extends to $2 n-1$, i.e., higher by one than the corresponding expansion for $F_{L}$ analysed in Ref. [34]. Thus the highest coefficients $d_{a, 2 n-1}^{(n)}$ at each order $n$ are identical also for $C_{1}$ and $C_{2}$. Recall that also the leading logarithms of the physical evolution kernels (3.26) to $\mathrm{N}^{3} \mathrm{LO}$ are the same for $a=1,2,3$. Their $1 / N$ contributions are

$$
\begin{aligned}
\left.K_{a, 1}\right|_{N^{-1} L} & =-2 \beta_{0} C_{F}-16 C_{F}^{2}, \\
\left.K_{a, 2}\right|_{N^{-1} L^{2}} & =-2 \beta_{0}^{2} C_{F}-24 \beta_{0} C_{F}^{2}, \\
\left.K_{a, 3}\right|_{N^{-1} L^{3}} & =-2 \beta_{0}^{3} C_{F}-\frac{88}{3} \beta_{0}^{2} C_{F}^{2} .
\end{aligned}
$$

As discussed at the end of Section 3, the vanishing of higher than single-enhanced logarithms in $K_{a, n}$ leads to relations between coefficient-function coefficients at different orders. For the two highest terms at all orders one finds

$$
\begin{aligned}
d_{a, 2 n-1}^{(n)} & =d_{a, 1}^{(1)} \frac{c_{12}^{n-1}}{(n-1) !} \equiv d_{11} \frac{c_{12}^{n-1}}{(n-1) !} \\
d_{a, 2 n-2}^{(n)} & =d_{11}\left\{c_{23}-c_{12} c_{11}\right\} \frac{\theta_{n 3} c_{12}^{n-3}}{(n-3) !}+\left\{d_{a, 2}^{(2)}-d_{a, 0}^{(1)} c_{12}\right\} \frac{\theta_{n 2} c_{12}^{n-2}}{(n-2) !}+d_{a, 0}^{(1)} \frac{c_{12}^{n-1}}{(n-1) !} \\
& =d_{11}\left\{h_{12} \frac{\theta_{n 3} c_{12}^{n-3}}{(n-3) !}+h_{21} \frac{\theta_{n 2} c_{12}^{n-2}}{(n-2) !}\right\}+d_{a, 0}^{(1)} \frac{c_{12}^{n-1}}{(n-1) !} .
\end{aligned}
$$

$d_{a, 1}^{(1)}$ is independent of $a$, as noted above, hence we denote this coefficient by $d_{11}$ below. $\theta_{k l}$ in (5.4) has been defined below Eq. (4.5), and the coefficients $h_{12}$ and $h_{21}$ in the last line are given by

$$
\begin{aligned}
& h_{12}=c_{23}-c_{12} c_{11}=\frac{1}{3} \beta_{0} c_{12}, \\
& d_{11} h_{21}=d_{a, 2}^{(2)}-d_{a, 0}^{(1)} c_{12} .
\end{aligned}
$$

Here the second identity in the first line arises from the soft-gluon exponentiation (4.1) together with the LL and NLL expansions (4.4) and (4.5). 
A comparison with the tower-expansion [51] of the soft-gluon resummation reveals that also Eqs. (5.3) and (5.4) correspond to an exponential structure which can be written as

$$
\begin{aligned}
& C_{a}(N)-\left.C_{a}\right|_{N^{0} L^{k}}= \\
& \quad \frac{1}{N}\left(\left[d_{11} L+d_{a, 0}^{(1)}\right] a_{\mathrm{s}}+\left[\widetilde{d}_{a, 1}^{(2)} L+d_{a, 0}^{(2)}\right] a_{\mathrm{s}}^{2}+\ldots\right) \exp \left\{L h_{1}\left(a_{\mathrm{s}} L\right)+h_{2}\left(a_{\mathrm{s}} L\right)+\ldots\right\},
\end{aligned}
$$

where also the functions $h_{k}$ are defined in terms of a power expansion,

$$
h_{k}\left(a_{\mathrm{s}} L\right) \equiv \sum_{k=1} h_{k n}\left(a_{\mathrm{s}} L\right)^{n}
$$

Notice that $\widetilde{d}_{a, 1}^{(2)}$ in Eq. (5.6) is not identical to $d_{a, 1}^{(2)}$ in Eq. (5.1) - the latter quantity receives a contribution from the expansion of the exponential. In this notation the third tower of logarithms is given by

$$
\begin{aligned}
d_{a, 2 n-3}^{(n)} & =d_{11}\left\{\frac{\theta_{n 3} h_{11}^{n-3}}{(n-3) !}\left(h_{22}+\frac{1}{2} h_{21}^{2}\right)+\frac{\theta_{n 4} h_{11}^{n-4}}{(n-4) !}\left(h_{13}+h_{12} h_{21}\right)+\frac{\theta_{n 5} g_{11}^{n-5}}{2(n-5) !} h_{12}^{2}\right\} \\
& +d_{a, 0}^{(1)}\left\{\frac{\theta_{n 2} h_{11}^{n-2}}{(n-2) !} h_{21}+\frac{\theta_{n 3} h_{11}^{n-3}}{(n-3) !} h_{12}\right\}+\widetilde{d}_{a, 1}^{(2)} \frac{\theta_{n 2} h_{11}^{n-2}}{(n-2) !}
\end{aligned}
$$

with $h_{11}=c_{12}=2 C_{F}$.

The new coefficient $h_{k n}$ entering Eq. (5.8) (and its lower-logarithmic generalizations) can be determined iteratively from fixed-order information. The exponentiation (5.6) then ensures the vanishing of the third-highest (and lower) double-logarithmic contributions to the physical kernel at all orders in $\alpha_{s}$. Consequently the conjectured single-logarithmic large- $x$ enhancement of the physical kernel is equivalent to an exponentiation in Mellin space beyond the leading $N^{0} L^{k}$ contributions.

All coefficients entering Eqs. (5.3), (5.4) and (5.8) can be determined from present information. The corresponding coefficients of the exponent turn out to be the same for $F_{1}$ and $F_{2}$. They read

$$
\begin{aligned}
& h_{1 k}=g_{1 k} \quad \text { for } \quad k=1,2,3, \\
& h_{21}=g_{21}+\frac{1}{2} \beta_{0}+6 C_{F}, \\
& h_{22}=g_{22}+\frac{5}{24} \beta_{0}^{2}+\frac{17}{9} \beta_{0} C_{F}-18 C_{F}^{2} .
\end{aligned}
$$

We conclude that the $1 / N$ leading-logarithmic function $h_{1}\left(a_{\mathrm{s}} L\right)$ for DIS is identical to its softgluon counterpart (4.4). The function $h_{2}\left(a_{\mathrm{s}} L\right)$, on the other hand, receives additional contributions which, it appears, prevent direct predictions of $g_{23}$ etc from Eqs. (5.10) and (5.11). This situation is analogous to that for $F_{L}$ found in Ref. [34]. Hence also here the present predictivity of the exponentiation is restricted to the three highest logarithms at all higher orders in $\alpha_{s}$.

The prefactor functions in Eq. (5.6) required to this accuracy are given by the coefficient

$$
d_{11}=2 C_{F},
$$


and for $\mathcal{F}_{1}-$ and $\mathcal{F}_{3}$, recall the discussion below Eq. (5.1) - by

$$
\begin{aligned}
d_{1,0}^{(1)}= & \frac{13}{2} C_{F}+2 \gamma_{\mathrm{e}} C_{F} \\
\widetilde{d}_{1,1}^{(2)}= & -C_{F}^{2}\left(47+4 \zeta_{2}-18 \gamma_{\mathrm{e}}-4 \gamma_{\mathrm{e}}^{2}\right)+C_{F} C_{A}\left(\frac{1133}{36}-4 \zeta_{2}+\frac{11}{3} \gamma_{\mathrm{e}}\right) \\
& -C_{F} n_{f}\left(\frac{127}{18}+\frac{2}{3} \gamma_{\mathrm{e}}\right) .
\end{aligned}
$$

The corresponding coefficients for $\mathcal{F}_{2}$ read

$$
\begin{aligned}
d_{2,0}^{(1)}= & \frac{21}{2} C_{F}+2 \gamma_{\mathrm{e}} C_{F} \\
\widetilde{d}_{2,1}^{(2)}= & -C_{F}^{2}\left(119-28 \zeta_{2}-18 \gamma_{\mathrm{e}}-4 \gamma_{\mathrm{e}}^{2}\right)+C_{F} C_{A}\left(\frac{1973}{36}-20 \zeta_{2}+\frac{11}{3} \gamma_{\mathrm{e}}\right) \\
& -C_{F} n_{f}\left(\frac{151}{18}+\frac{2}{3} \gamma_{\mathrm{e}}\right) .
\end{aligned}
$$

Insertion of Eqs. (5.9) - (5.16) into Eqs. (5.3), (5.4) and (5.8) provides explicit formulae for the coefficients of the three highest $1 / N$ logarithms in Eq. (5.1) at all orders in $\alpha_{\mathrm{s}}$. For brevity, we here only present the fourth-order results, Mellin-inverted back to $x$-space. For $\mathcal{F}_{1}$ one obtains

$$
\begin{aligned}
c_{1,4}(x)= & \left.c_{1,4}\right|_{\mathcal{D}_{k}, \delta(1-x)}-\frac{16}{3} C_{F}^{4} L_{x}^{7}+\left\{\frac{232}{3} C_{F}^{4}+\frac{28}{3} C_{F}^{3} \beta_{0}\right\} L_{x}^{6} \\
& -\left\{\left[188-128 \zeta_{2}\right] C_{F}^{4}+\left[12-48 \zeta_{2}\right] C_{F}^{3} C_{A}+\frac{1460}{9} C_{F}^{3} \beta_{0}+\frac{16}{3} C_{F}^{2} \beta_{0}^{2}\right\} L_{x}^{5} \\
& +O\left(L_{x}^{4}\right),
\end{aligned}
$$

where we have used the abbreviations

$$
\mathcal{D}_{n} \equiv\left[(1-x)^{-1} \ln ^{n}(1-x)\right]_{+} \quad \text { and } \quad L_{x} \equiv \ln (1-x) .
$$

The coefficients of $C_{F}^{4} L_{x}^{7}, C_{F}^{3} \beta_{0} L_{x}^{6}$ and $C_{F}^{2} \beta_{0}^{2} L_{x}^{5}$ in Eq. (5.17) are the negative of those of the corresponding +-distributions given (in terms of $C_{F}, C_{A}$ and $n_{f}$ ) in Eqs. (5.4) - (5.6) of Ref. [12]. Hence the general pattern noted below Eq. (3.4) is part of the present exponentiation and predicted to persist to higher orders. The corresponding result for $\mathcal{F}_{2}$ can be written as

$$
\begin{aligned}
c_{2,4}(x)= & c_{1,4}(x)+\frac{16}{3} C_{F}^{4} L_{x}^{6} \\
& +\left\{\left[72-64 \zeta_{2}\right] C_{F}^{4}-32\left[1-\zeta_{2}\right] C_{F}^{3} C_{A}-\frac{40}{3} C_{F}^{3} \beta_{0}\right\} L_{x}^{5}+O\left(L_{x}^{4}\right) .
\end{aligned}
$$

This result, obtained from the subleading terms of the physical kernels of $F_{1}$ and $F_{2}$, is consistent with Eq. (16) (which also provides the coefficient of $L_{x}^{4}$ ) of Ref. [34], derived from the leading large- $x$ physical kernel of the longitudinal structure function $F_{L}$. This agreement provides a rather non-trivial confirmation of our approach. 
Although it is not fully known at present, it is instructive to consider also the fourth tower of logarithms. The corresponding generalization of Eq. (16) of [51] to the present case (5.1) reads

$$
\begin{aligned}
d_{a, 2 n-4}^{(n)}= & d_{11}\left\{\frac{\theta_{n 3} h_{11}^{n-3}}{(n-3) !} h_{a, 3}^{(1)}+\frac{\theta_{n 4} h_{11}^{n-4}}{(n-4) !}\left(h_{23}+h_{22} h_{21}+\frac{1}{6} h_{21}^{3}\right)+\frac{\theta_{n 7} h_{11}^{n-7}}{6(n-7) !} h_{12}^{3}\right. \\
& \left.+\frac{\theta_{n 5} h_{11}^{n-5}}{(n-5) !}\left(h_{14}+h_{13} h_{21}+h_{12} h_{22}+\frac{1}{2} h_{12} h_{21}^{2}\right)+\frac{\theta_{n 6} h_{11}^{n-6}}{(n-6) !}\left(h_{13} h_{12}+\frac{1}{2} h_{12}^{2} h_{21}\right)\right\} \\
+ & d_{a, 0}^{(1)}\left\{\frac{\theta_{n 3} h_{11}^{n-3}}{(n-3) !}\left(h_{22}+\frac{1}{2} h_{21}^{2}\right)+\frac{\theta_{n 4} h_{11}^{n-4}}{(n-4) !}\left(h_{13}+h_{12} h_{21}\right)+\frac{\theta_{n 5} g_{11}^{n-5}}{2(n-5) !} h_{12}^{2}\right\} \\
+ & \widetilde{d}_{a, 1}^{(2)}\left\{\frac{\theta_{n 3} h_{11}^{n-3}}{(n-3) !} h_{21}+\frac{\theta_{n 4} h_{11}^{n-4}}{(n-4) !} h_{12}\right\}+d_{a, 0}^{(2)} \frac{\theta_{n 2} h_{11}^{n-2}}{(n-2) !} .
\end{aligned}
$$

The additional second- and third-order coefficients in Eq. (5.19) are

$$
\begin{aligned}
d_{1,0}^{(2)}= & -C_{F}^{2}\left(\frac{295}{4}+7 \zeta_{2}-12 \zeta_{3}-\frac{23}{2} \gamma_{\mathrm{e}}+4 \zeta_{2} \gamma_{\mathrm{e}}-31 \gamma_{\mathrm{e}}^{2}-4 \gamma_{\mathrm{e}}^{3}\right) \\
& +C_{F} C_{A}\left(\frac{12419}{108}-\frac{35}{3} \zeta_{2}-20 \zeta_{3}+\frac{781}{18} \gamma_{\mathrm{e}}-4 \zeta_{2} \gamma_{\mathrm{e}}+\frac{11}{3} \gamma_{\mathrm{e}}^{2}\right) \\
& -C_{F} n_{f}\left(\frac{1243}{54}-\frac{2}{3} \zeta_{2}+\frac{83}{9} \gamma_{\mathrm{e}}+\frac{2}{3} \gamma_{\mathrm{e}}^{2}\right), \\
d_{2,0}^{(2)}= & -C_{F}^{2}\left(\frac{431}{4}+47 \zeta_{2}-60 \zeta_{3}+\frac{49}{2} \gamma_{\mathrm{e}}-28 \zeta_{2} \gamma_{\mathrm{e}}-39 \gamma_{\mathrm{e}}^{2}-4 \gamma_{\mathrm{e}}^{3}\right) \\
& +C_{F} C_{A}\left(\frac{17579}{108}+\frac{13}{3} \zeta_{2}-44 \zeta_{3}+\frac{1333}{18} \gamma_{\mathrm{e}}-20 \zeta_{2} \gamma_{\mathrm{e}}+\frac{11}{3} \gamma_{\mathrm{e}}^{2}\right) \\
& -C_{F} n_{f}\left(\frac{1699}{54}-\frac{2}{3} \zeta_{2}+\frac{107}{9} \gamma_{\mathrm{e}}+\frac{2}{3} \gamma_{\mathrm{e}}^{2}\right)
\end{aligned}
$$

and

$$
\begin{aligned}
h_{1,3}^{(1)}= & g_{31}+C_{F}^{2}\left(160-\frac{88}{3} \zeta_{2}-36 \gamma_{\mathrm{e}}\right)-C_{F} \beta_{0}\left(\frac{116}{9}+2 \zeta_{2}-\frac{34}{9} \gamma_{\mathrm{e}}\right)+\beta_{0}^{2}\left(\frac{51}{16}+\frac{5}{12} \gamma_{\mathrm{e}}\right) \\
& +\left(C_{A}-2 C_{F}\right)\left\{C_{F}\left(\frac{211}{6}-\frac{44}{3} \zeta_{2}\right)+C_{A}\left(\frac{13}{3}-\frac{5}{3} \zeta_{2}\right)-\beta_{0}\left(\frac{11}{6}+\zeta_{2}\right)\right\} \\
h_{2,3}^{(1)}= & h_{1,3}^{(1)}+136 C_{F}^{2}-\frac{160}{9} C_{F} \beta_{0}+\frac{5}{6} \beta_{0}^{2} \\
& -\left(C_{A}-2 C_{F}\right)\left\{\left(80 C_{F}-8 \beta_{0}\right)\left(1-\zeta_{2}\right)+16\left(C_{A}-2 C_{F}\right)\left(\zeta_{3}-\zeta_{2}\right)\right\}
\end{aligned}
$$

with $g_{31}$ given in Eq. (4.9). Thus, in contrast to Eqs. (5.10) and (5.11), the NNLL resummation functions $h_{a, 3}$ are not the same for $a=1$ and $a=2$, and the deviation of their leading coefficient from $g_{31}$ involves $\zeta$-functions, including $\zeta_{3}$ in the case of $\mathcal{F}_{2}$. 
The only other new coefficient entering Eq. (5.19) at order $\alpha_{\mathrm{s}}^{4}$ is $h_{23}$. This quantity can be constrained, but not completely fixed, from the rather obvious extension of Eqs. (5.2) for the ( $a$-independent) leading $1 / N$ behaviour of the physical kernel to the next order,

$$
\left.K_{a, 4}\right|_{N^{-1} L^{4}}=-2 \beta_{0}^{4} C_{F}-\xi_{\mathrm{DIS}_{4}} \beta_{0}^{3} C_{F}^{2} .
$$

The first term on the right-hand-side is fixed by the all-order leading- $n_{f}$ result for $C_{a}$ [36]. Moreover the all- $x$ expressions (3.26) strongly suggest that terms with a lower power of $\beta_{0}$ only contribute to $K_{a, 4}(N)$ at higher orders in $1 / N$. The consistency of Eqs. (5.19) - (5.24) then requires

$$
h_{23}=g_{23}+\frac{1}{8} \beta_{0}^{3}+\left(\frac{\xi_{\mathrm{DIS}_{4}}}{8}-\frac{53}{18}\right) \beta_{0}^{2} C_{F}-\frac{34}{3} \beta_{0} C_{F}^{2}+72 C_{F}^{3} .
$$

As implied by the notation used above, also this coefficient of the NLL resummation function is the same for all structure functions (2.1). The missing information for $\xi_{\mathrm{DIS}_{4}}$ is a next-to-leading large- $n_{f}$ contribution to the fourth-order coefficient function. Since the leading large- $n_{f}$ terms were derived more than ten years ago, and enormous calculational progress has been made in this time, an extension to the next order in $n_{f}$ should be feasible in the near future. We will comment on relations between the rational coefficients in Eqs. (5.10), (5.11) and (5.25) below Eq. (6.21).

The resulting next contribution to Eq. (5.17) reads (recall $L_{x} \equiv \ln (1-x)$ )

$$
\begin{aligned}
\left.c_{1,4}\right|_{L_{x}^{4}} & =-C_{F}^{4}\left(\frac{1270}{3}+1424 \zeta_{2}+\frac{400}{3} \zeta_{3}\right)+C_{F}^{3} C_{A}\left(\frac{1576}{9}-\frac{1312}{3} \zeta_{2}-400 \zeta_{3}\right) \\
& +C_{F}^{3} \beta_{0}\left(\frac{7583}{9}-\frac{520}{3} \zeta_{2}\right)-C_{F}^{2} C_{A}^{2}\left(\frac{46}{3}+\frac{20}{3} \zeta_{2}\right)+C_{F}^{2} C_{A} \beta_{0}\left(\frac{70}{3}-40 \zeta_{2}\right) \\
& +C_{F}^{2} \beta_{0}^{2}\left(\frac{\xi_{\mathrm{DIS}_{4}}}{4}+\frac{277}{3}\right)+C_{F} \beta_{0}^{3} .
\end{aligned}
$$

As expected, the coefficient of $C_{F} \beta_{0}^{3}$ is the negative of the corresponding coefficient in Eqs. (5.7) of Ref. [12]. The presumed $a$-independent of $\xi_{\mathrm{DIS}_{4}}$ leads to a definite prediction for the $\ln ^{4}(1-x)$ term of the fourth-order longitudinal structure function,

$$
\begin{aligned}
\left.c_{2,4}\right|_{L_{x}^{4}} & =\left.c_{1,4}\right|_{L_{x}^{4}}+C_{F}^{4}\left(32 \zeta_{2}-160 \zeta_{3}\right)-C_{F}^{3} C_{A}\left(8+224 \zeta_{2}-208 \zeta_{3}\right)+12 C_{F}^{2} \beta_{0}^{2} \\
& -C_{F}^{3} \beta_{0}\left(80-\frac{352}{3} \zeta_{2}\right)+64 C_{F}^{2} C_{A}^{2}\left(\zeta_{2}-\zeta_{3}\right)+\frac{176}{3} C_{F}^{2} C_{A} \beta_{0}\left(1-\zeta_{2}\right) .
\end{aligned}
$$

This result completes the independent re-derivation of Eq. (16) in Ref. [34].

The vanishing of the double-logarithmic $N^{-1} \ln ^{6} N$ contribution to $K_{a, 5}(N)$ fixes the final coefficient in Eq. (5.19),

$$
h_{14}=\frac{1}{5} C_{F} \beta_{0}^{3}=g_{14}
$$

where the second equality refers to Eq. (4.4). Thus, up to the presently unknown number $\xi_{\mathrm{DIS}_{4}}$, the four highest $1 / N$ (or $\left.(1-x)^{0}\right)$ logarithms for the structure functions (2.1) are fixed to all orders in $\alpha_{\mathrm{s}}$. Moreover it appears obvious from Eqs. (5.9) and (5.28) that $h_{1}\left(a_{\mathrm{s}} L\right)$ is identical to its soft-gluon counterpart $g_{1}\left(a_{\mathrm{s}} L\right)$. 
Instead of working out the corresponding all-order $N$-space formalism at the next power(s) in $1 / N$, we close this section on deep-inelastic scattering by presenting the fourth-order extension of Eqs. (3.6) - (3.14), recall the last paragraph of Section 3:

$$
\begin{aligned}
& c_{1,4}(x)=\left(\ln ^{7}(1-x) 8 / 3 C_{F}^{4}-\ln ^{6}(1-x) 14 / 3 C_{F}^{3} \beta_{0}+\ln ^{5}(1-x) 8 / 3 C_{F}^{2} \beta_{0}^{2}\right) p_{q q}(x) \\
& +\ln ^{6}(1-x)\left[C_{F}^{4}\left\{p_{q q}(x)\left(-14-68 / 3 \mathrm{H}_{0}\right)+4+8 \mathrm{H}_{0}-(1-x)\left(6+4 \mathrm{H}_{0}\right)\right\}\right] \\
& +\ln ^{5}(1-x)\left[C _ { F } ^ { 4 } \left\{p_{q q}(x)\left(-9-8 \widetilde{\mathrm{H}}_{1,0}+448 / 3 \mathrm{H}_{0,0}+84 \mathrm{H}_{0}-64 \zeta_{2}\right)+48 \widetilde{\mathrm{H}}_{1,0}\right.\right. \\
& \left.-22-96 \mathrm{H}_{0,0}-104 \mathrm{H}_{0}-(1-x)\left(13+24 \widetilde{\mathrm{H}}_{1,0}-48 \mathrm{H}_{0,0}-84 \mathrm{H}_{0}-16 \zeta_{2}\right)\right\} \\
& +C_{F}^{3} \beta_{0}\left\{p_{q q}(x)\left(41+316 / 9 \mathrm{H}_{0}\right)-10-32 / 3 \mathrm{H}_{0}+(1-x)\left(41 / 3+16 / 3 \mathrm{H}_{0}\right)\right\} \\
& +C_{F}^{3} C_{A}\left\{p_{q q}(x)\left(16+8 \widetilde{\mathrm{H}}_{1,0}+8 \mathrm{H}_{0,0}-24 \zeta_{2}\right)+4+(1-x)\left(28-8 \zeta_{2}\right)\right\} \\
& \left.+C_{F}^{3}\left(C_{A}-2 C_{F}\right) p_{q q}(-x)\left(16 \widetilde{\mathrm{H}}_{-1,0}-8 \mathrm{H}_{0,0}\right)\right] \\
& +o\left(\ln ^{4}(1-x)\right) \text {, } \\
& c_{2,4}(x)=c_{1,4}(x)+\ln ^{6}(1-x) 16 / 3 x C_{F}^{4} \\
& +\ln ^{5}(1-x)\left[C_{F}^{4}\left\{16-x\left(8+48 \mathrm{H}_{0}\right)\right\}-40 / 3 x C_{F}^{3} \beta_{0}-C_{F}^{3}\left(C_{A}-2 C_{F}\right) 32 x\left(1-\zeta_{2}\right)\right] \\
& +\ln ^{4}(1-x)\left[C_{F}^{4}\left\{8-80 \mathrm{H}_{0}-\left(24+48 \widetilde{\mathrm{H}}_{1,0}-288 \mathrm{H}_{0,0}-48 \mathrm{H}_{0}+160 \zeta_{2}\right) x\right\}\right. \\
& -C_{F}^{3} \beta_{0}\left\{112 / 3-\left(224 / 3+104 \mathrm{H}_{0}\right) x\right\}+C_{F}^{2} \beta_{0}^{2} 12 x \\
& +C_{F}^{3}\left(C_{A}-2 C_{F}\right)\left\{-64 /\left(5 x^{2}\right)\left(\widetilde{\mathrm{H}}_{-1,0}-\zeta_{2} / 2\right)-64 /(5 x)\left(1-\mathrm{H}_{0}\right)-304 / 5\right. \\
& -64 \widetilde{\mathrm{H}}_{-1,0}-32 / 5 \mathrm{H}_{0}+96 \zeta_{2}+x\left(424 / 5+32\left[2 \widetilde{\mathrm{H}}_{-1,-1,0}-\widetilde{\mathrm{H}}_{-1,0,0}\right.\right. \\
& \left.\left.+\widetilde{\mathrm{H}}_{1,0,0}-\widetilde{\mathrm{H}}_{-1,0}+\mathrm{H}_{0,0}\right]+1168 / 5 \mathrm{H}_{0}-80 \zeta_{2}-192 \zeta_{2} \mathrm{H}_{0}-48 \zeta_{3}\right) \\
& \left.-96 / 5 x^{2}\left(1+\mathrm{H}_{0}\right)+96 / 5 x^{3}\left(\widetilde{\mathrm{H}}_{-1,0}-\mathrm{H}_{0,0}+\zeta_{2} / 2\right)\right\} \\
& \left.+C_{F}^{2}\left(C_{A}-2 C_{F}\right) \beta_{0} 176 / 3 x\left(1-\zeta_{2}\right)+C_{F}^{2}\left(C_{A}-2 C_{F}\right)^{2} 64 x\left(\zeta_{2}-\zeta_{3}\right)\right] \\
& +o\left(\ln ^{3}(1-x)\right) \text {, } \\
& c_{3,4}(x)=c_{1,4}(x)-\ln ^{6}(1-x) 8 / 3 C_{F}^{4}(1-x) \\
& +\ln ^{5}(1-x)\left[C_{F}^{4}\left\{-64 \mathrm{H}_{0}+(1-x)\left(36+56 \mathrm{H}_{0}-32 \zeta_{2}\right)\right\}\right. \\
& +20 / 3 C_{F}^{3} \beta_{0}(1-x)+C_{F}^{3} C_{A}\left\{32 \mathrm{H}_{0}-16(1-x)\left(1+\mathrm{H}_{0}-\zeta_{2}\right)\right\} \\
& \left.-C_{F}^{3}\left(C_{A}-2 C_{F}\right) 16 p_{q q}(-x)\left(2 \widetilde{\mathrm{H}}_{-1,0}-\mathrm{H}_{0,0}\right)\right] \\
& +o\left(\ln ^{4}(1-x)\right) \text {. }
\end{aligned}
$$

The $\ln ^{4}(1-x)$ contribution to $c_{1,4}$ involves two unknown coefficients of $K_{a, 4}(x)$, see Eqs. (3.26) and (5.24). The corresponding terms in Eq. (5.31) can be predicted completely. However, as $C_{3}-C_{1}$ does not correspond to an observable, we have refrained from writing them down here. 


\section{Results for fragmentation and the Drell-Yan process}

As discussed above, the subleading large- $x / \operatorname{large}-N$ structure of the coefficient functions for the fragmentation functions (2.10) in semi-inclusive $e^{+} e^{-}$annihilation (SIA) is completely analogous to that of their DIS counterparts addressed in the previous section. Consequently the notation (5.1) can be used for the present $1 / N$ coefficients as well. Also these contributions can be resummed in the form (5.6), with the first four towers of logarithms given by Eqs. (5.3) - (5.8) and (5.19).

The coefficient functions $C_{T}$ and $C_{A}$ are identical up to terms of order $1 / N^{2}$ or $(1-x)$, cf. Eqs. (3.21) - (3.23) above. The leading $1 / N$ logarithms of the physical kernels (2.8) are the same for all three fragmentation functions $\mathcal{F}_{I} \equiv \mathcal{F}_{T}+\mathcal{F}_{L}, \mathcal{F}_{T}$ and $\mathcal{F}_{A}($ recall $L \equiv \ln N)$,

$$
\begin{aligned}
\left.K_{a, 1}\right|_{N^{-1} L} & =-2 \beta_{0} C_{F}+16 C_{F}^{2}, \\
\left.K_{a, 2}\right|_{N^{-1} L^{2}} & =-2 \beta_{0}^{2} C_{F}+24 \beta_{0} C_{F}^{2}, \\
\left.K_{a, 3}\right|_{N^{-1} L^{3}} & =-2 \beta_{0}^{3} C_{F}+\frac{88}{3} \beta_{0}^{2} C_{F}^{2}, \\
\left.K_{a, 4}\right|_{N^{-1} L^{4}} & =-2 \beta_{0}^{4} C_{F}+\xi_{\mathrm{SIA}_{4}} \beta_{0}^{3} C_{F}^{2} .
\end{aligned}
$$

The first three lines derive from Eq. (3.26). These results are identical to Eqs. (5.2) for the DIS kernels except for the different sign of the non-leading large- $n_{f}$ terms. The close relation between the SIA and DIS cases suggests $\xi_{\mathrm{SIA}_{4}}=\xi_{\mathrm{DIS}_{4}}$ for the fourth-order generalization in the final line corresponding to Eq. (5.24).

The expansion coefficients of the LL and NLL contributions to the resummation exponential (5.6), fixed by Eqs. (6.1) and the vanishing of higher than single-logarithmic contributions, read

$$
\begin{aligned}
& h_{1 k}=g_{1 k} \quad \text { for } \quad k=1, \ldots, 4, \\
& h_{21}=g_{21}+\frac{1}{2} \beta_{0}-6 C_{F}, \\
& h_{22}=g_{22}+\frac{5}{24} \beta_{0}^{2}-\frac{17}{9} \beta_{0} C_{F}-18 C_{F}^{2}, \\
& h_{23}=g_{23}+\frac{1}{8} \beta_{0}^{3}+\left(\frac{53}{18}-\frac{\xi_{\mathrm{SIA}_{4}}}{8}\right) \beta_{0}^{2} C_{F}-\frac{34}{3} \beta_{0} C_{F}^{2}-72 C_{F}^{3} .
\end{aligned}
$$

The coefficients (6.3) - (6.5) differ from their DIS counterparts (5.10), (5.11) and (5.25) only by the signs of every second term in the expansion in powers of $\beta_{0}$. The first coefficients of the NNLL resummation function $h_{a, 3}$ (defined as $g_{3}$ in Eq. (4.1)), on the other hand, are neither the same for the coefficient functions $C_{T, A}$ and $C_{I}$, the SIA analogue of $C_{2}$, nor do they show a close relation to their DIS counterparts (5.22) and (5.23). These coefficients are

$$
\begin{aligned}
h_{T, 3}^{(1)}= & g_{31}-C_{F}^{2}\left(240-\frac{88}{3} \zeta_{2}+36 \gamma_{\mathrm{e}}\right)-C_{F} \beta_{0}\left(\frac{139}{9}+2 \zeta_{2}+\frac{34}{9} \gamma_{\mathrm{e}}\right)-\beta_{0}^{2}\left(\frac{9}{16}-\frac{5}{12} \gamma_{\mathrm{e}}\right) \\
& -\left(C_{A}-2 C_{F}\right)\left\{C_{F}\left(\frac{1}{6}-\frac{44}{3} \zeta_{2}\right)+C_{A}\left(\frac{34}{3}-\frac{5}{3} \zeta_{2}\right)-\beta_{0}\left(\frac{49}{6}-\zeta_{2}\right)\right\}
\end{aligned}
$$




$$
\begin{aligned}
h_{I, 3}^{(1)}= & h_{T, 3}^{(1)}+20 C_{F}^{2}+\frac{8}{9} C_{F} \beta_{0}+\frac{5}{12} \beta_{0}^{2} \\
& +\left(C_{A}-2 C_{F}\right)\left\{\left(8 C_{F}+4 \beta_{0}\right)\left(1-\zeta_{2}\right)-8\left(C_{A}-2 C_{F}\right)\left(\zeta_{3}-\zeta_{2}\right)\right\} .
\end{aligned}
$$

Finally the required coefficients of the prefactors of the exponential, again obtained by expanding Eq. (5.6) in powers of $\alpha_{\mathrm{s}}$ and comparing to the results in Section 3, are given by

$$
d_{11}=2 C_{F}
$$

for both coefficient functions, the same result as in Eq. (5.12) for the DIS case,

$$
\begin{aligned}
d_{T, 0}^{(1)}= & -\frac{23}{2} C_{F}+2 \gamma_{\mathrm{e}} C_{F} \\
\widetilde{d}_{T, 1}^{(2)}= & -C_{F}^{2}\left(97-20 \zeta_{2}+6 \gamma_{\mathrm{e}}-4 \gamma_{\mathrm{e}}^{2}\right)+C_{F} C_{A}\left(\frac{665}{36}-4 \zeta_{2}+\frac{11}{3} \gamma_{\mathrm{e}}\right) \\
& -C_{F} n_{f}\left(\frac{19}{18}+\frac{2}{3} \gamma_{\mathrm{e}}\right) \\
d_{T, 0}^{(2)}= & C_{F}^{2}\left(\frac{481}{4}-157 \zeta_{2}+12 \zeta_{3}-\frac{125}{2} \gamma_{\mathrm{e}}+20 \zeta_{2} \gamma_{\mathrm{e}}-29 \gamma_{\mathrm{e}}^{2}+4 \gamma_{\mathrm{e}}^{3}\right) \\
& -C_{F} C_{A}\left(\frac{9325}{108}-\frac{37}{3} \zeta_{2}+20 \zeta_{3}+\frac{47}{18} \gamma_{\mathrm{e}}+4 \zeta_{2} \gamma_{\mathrm{e}}-\frac{11}{3} \gamma_{\mathrm{e}}^{2}\right) \\
& +C_{F} n_{f}\left(\frac{989}{54}+\frac{2}{3} \zeta_{2}+\frac{25}{9} \gamma_{\mathrm{e}}-\frac{2}{3} \gamma_{\mathrm{e}}^{2}\right)
\end{aligned}
$$

and

$$
\begin{aligned}
d_{I, 0}^{(1)}= & -\frac{19}{2} C_{F}+2 \gamma_{\mathrm{e}} C_{F} \\
\widetilde{d}_{I, 1}^{(2)}= & -C_{F}^{2}\left(109-36 \zeta_{2}+6 \gamma_{\mathrm{e}}-4 \gamma_{\mathrm{e}}^{2}\right)+C_{F} C_{A}\left(\frac{1085}{36}-12 \zeta_{2}+\frac{11}{3} \gamma_{\mathrm{e}}\right) \\
& -C_{F} n_{f}\left(\frac{31}{18}+\frac{2}{3} \gamma_{\mathrm{e}}\right) \\
d_{I, 0}^{(2)}= & C_{F}^{2}\left(\frac{413}{4}-153 \zeta_{2}+36 \zeta_{3}-\frac{161}{2} \gamma_{\mathrm{e}}+36 \zeta_{2} \gamma_{\mathrm{e}}-25 \gamma_{\mathrm{e}}^{2}+4 \gamma_{\mathrm{e}}^{3}\right) \\
& -C_{F} C_{A}\left(\frac{6745}{108}-\frac{61}{3} \zeta_{2}+32 \zeta_{3}-\frac{229}{18} \gamma_{\mathrm{e}}+12 \zeta_{2} \gamma_{\mathrm{e}}-\frac{11}{3} \gamma_{\mathrm{e}}^{2}\right) \\
& +C_{F} n_{f}\left(\frac{761}{54}+\frac{2}{3} \zeta_{2}+\frac{13}{9} \gamma_{\mathrm{e}}-\frac{2}{3} \gamma_{\mathrm{e}}^{2}\right) .
\end{aligned}
$$

Except for the coefficients with $\zeta_{3}$ (and some obvious terms with $\gamma_{\mathrm{e}}$ ) there is no direct relation either between Eqs. (6.9) - (6.14) and their DIS counterparts (5.12) - (5.16), (5.20) and (5.21).

Inserting Eqs. (6.2) - (6.14) into Eqs. (5.3) - (5.8) and (5.19) we arrive at explicit predictions for the coefficients of the four highest $1 / N$ logarithms to all orders in $\alpha_{\mathrm{s}}$, with the fourth logarithm including the unknown coefficient $\xi_{\mathrm{SIA}_{4}}$ of Eq. (6.5). After Mellin inversion back to $x$-space the fourth-order result for $\mathcal{F}_{T}$ (and $\mathcal{F}_{A}$, see above) read 


$$
\begin{aligned}
c_{T, 4}(x)= & \left.c_{T, 4}\right|_{\mathcal{D}_{k}, \delta(1-x)}-\frac{16}{3} C_{F}^{4} L_{x}^{7}+\left\{\frac{16}{3} C_{F}^{4}+\frac{28}{3} C_{F}^{3} \beta_{0}\right\} L_{x}^{6} \\
+ & \left\{\left[104+32 \zeta_{2}\right] C_{F}^{4}-\left[52-48 \zeta_{2}\right] C_{F}^{3} C_{A}-\frac{424}{9} C_{F}^{3} \beta_{0}-\frac{16}{3} C_{F}^{2} \beta_{0}^{2}\right\} L_{x}^{5} \\
+ & \left\{C_{F}^{4}\left(-\frac{44}{3}-272 \zeta_{2}-\frac{400}{3} \zeta_{3}\right)+C_{F}^{3} C_{A}\left(\frac{964}{9}+\frac{112}{3} \zeta_{2}-400 \zeta_{3}\right)\right. \\
& -C_{F}^{3} \beta_{0}\left(\frac{223}{9}+\frac{280}{3} \zeta_{2}\right)-C_{F}^{2} C_{A}^{2}\left(78-\frac{20}{3} \zeta_{2}\right)+C_{F}^{2} C_{A} \beta_{0}\left(\frac{290}{3}-40 \zeta_{2}\right) \\
& \left.+C_{F}^{2} \beta_{0}^{2}\left(\frac{115}{3}-\frac{\xi_{\mathrm{SIA}_{4}}}{4}\right)+C_{F} \beta_{0}^{3}\right\} L_{x}^{4}+O\left(L_{x}^{3}\right),
\end{aligned}
$$

where we have again used the abbreviations introduced below Eq. (5.17). As for the corresponding +-distributions, see Ref. [17], the coefficients of $C_{F}^{4} L_{x}^{7}, C_{F}^{3} \beta_{0} L_{x}^{6}, C_{F}^{2} \beta_{0}^{2} L_{x}^{5}$ and $C_{F} \beta_{0}^{3} L_{x}^{4}$ in Eq. (6.15) are the same as in Eqs. (5.17) and (5.26) for the DIS case.

The corresponding predictions for the total fragmentation function $\mathcal{F}_{I}=\mathcal{F}_{T}+\mathcal{F}_{L}$ lead to the following results for the longitudinal fragmentation function $\mathcal{F}_{L}$ :

$$
\begin{aligned}
c_{L, 4}(x)= & \frac{8}{3} C_{F}^{4} L_{x}^{6}+\left\{\left[36-32 \zeta_{2}\right] C_{F}^{4}-16\left[1-\zeta_{2}\right] C_{F}^{3} C_{A}-\frac{20}{3} C_{F}^{3} \beta_{0}\right\} L_{x}^{5} \\
+ & \left\{C_{F}^{4}\left(64 \zeta_{2}-80 \zeta_{3}\right)-C_{F}^{3} C_{A}\left(4+112 \zeta_{2}-104 \zeta_{3}\right)-C_{F}^{3} \beta_{0}\left(40-\frac{176}{3} \zeta_{2}\right)\right. \\
& \left.+6 C_{F}^{2} \beta_{0}^{2}+32 C_{F}^{2} C_{A}^{2}\left(\zeta_{2}-\zeta_{3}\right)+\frac{88}{3} C_{F}^{2} C_{A} \beta_{0}\left(1-\zeta_{2}\right)\right\} L_{x}^{4}+O\left(L_{x}^{3}\right) .
\end{aligned}
$$

Besides an overall factor of two arising from the different definitions of $\mathcal{F}_{L}$ in SIA and DIS, this expression differs from its counterparts (5.18) and (5.27) for the longitudinal structure functions in DIS only in the coefficient of $\zeta_{2} C_{F}^{4} \ln ^{4}(1-x)$. Eq. (6.16) can be derived also via the physical evolution kernel for the longitudinal fragmentation function, in complete analogy with the DIS case in Ref. [34]. In fact, Eqs. (20) - (22) of that article hold for the present case as well, with the above difference arising from the second-order prefactor to the resummation exponential. This close relation between the spacelike and timelike cases does not persist at higher orders in $(1-x)$, as can be seen already by comparing Eqs. (3.9) and (3.18).

We now turn to the corresponding results for the non-singlet Drell-Yan cross section (2.11). The leading $1 / N$ contributions to its physical kernel are given by $\mathrm{G}$

$$
\begin{aligned}
\left.K_{\mathrm{DY}, 1}\right|_{N^{-1} L} & =-8 \beta_{0} C_{F}-32 C_{F}^{2}, \\
\left.K_{\mathrm{DY}, 2}\right|_{N^{-1} L^{2}} & =-16 \beta_{0}^{2} C_{F}-112 \beta_{0} C_{F}^{2}, \\
\left.K_{\mathrm{DY}, 3}\right|_{N^{-1} L^{3}} & =-32 \beta_{0}^{3} C_{F}+\xi_{\mathrm{DY}} \beta_{0}^{2} C_{F}^{2}, \\
\left.K_{\mathrm{DY}, 4}\right|_{N^{-1} L^{4}} & =-64 \beta_{0}^{4} C_{F}+\xi_{\mathrm{DY}} \beta_{0}^{3} C_{F}^{2} .
\end{aligned}
$$

Here the first two lines follow from Eqs. (3.27), while the third and the fourth are the obvious generalization to order $\alpha_{\mathrm{s}}^{4}$ and $\alpha_{\mathrm{s}}^{5}$, respectively, exploiting the complete analogy to the DIS and 
SIA cases discussed above. Also these parts of Eqs. (6.17) are of some interest despite the unknown subleading large- $\beta_{0}$ terms.

This can be seen from the resulting coefficients of the LL and NLL resummation exponents,

$$
\begin{aligned}
& h_{1 k}=g_{1 k} \quad \text { for } \quad k=1, \ldots, 4, \\
& h_{21}=g_{21}+\beta_{0}+7 C_{F}, \\
& h_{22}=g_{22}+\frac{5}{6} \beta_{0}^{2}-\left(7-\frac{\xi_{\mathrm{DY}_{3}}}{24}\right) \beta_{0} C_{F}-\frac{49}{2} C_{F}^{2}, \\
& h_{23}=g_{23}+\beta_{0}^{3}-\left(\frac{7}{3}+\frac{\xi_{\mathrm{DY}_{3}}}{24}-\frac{\xi_{\mathrm{DY}_{4}}}{32}\right) \beta_{0}^{2} C_{F}-\left(49-\frac{7 \xi_{\mathrm{DY}_{3}}}{24}\right) \beta_{0} C_{F}^{2}+\frac{343}{3} C_{F}^{3} .
\end{aligned}
$$

We note that, both here and in Eqs. (5.11) and (5.25) for the structure functions in DIS, the coefficients of $C_{F}^{n}$ in $h_{2 n}$ are given by $1 / n$ times the $n$-th power of the corresponding coefficient in $h_{21}$. Furthermore the coefficients of $\beta_{0} C_{F}^{2}$ in Eqs. (5.25) and (6.21) are the products of the respective $C_{F}$ and $\beta_{0} C_{F}$ coefficients in $h_{21}$ and $h_{22}$. These relations seem to point towards a general structure for the functions $h_{2}\left(a_{\mathrm{s}} L\right)$ in Eq. (5.6) which, we hope, can be uncovered in some more deductive approach to the $1 / N$ contributions to the coefficient functions.

The prefactor coefficients relevant for the highest three logarithms read

$$
\begin{aligned}
d_{\mathrm{DY}, 1}^{(1)} \equiv & d_{11}=8 C_{F}, \quad d_{\mathrm{DY}, 0}^{(1)}=8 \gamma_{\mathrm{e}} C_{F}, \\
\widetilde{d}_{\mathrm{DY}, 1}^{(2)}= & -C_{F}^{2}\left(156-128 \zeta_{2}-56 \gamma_{\mathrm{e}}-64 \gamma_{\mathrm{e}}^{2}\right)+C_{F} C_{A}\left(\frac{884}{9}-16 \zeta_{2}+\frac{88}{3} \gamma_{\mathrm{e}}\right) \\
& -C_{F} n_{f}\left(\frac{176}{9}+\frac{16}{3} \gamma_{\mathrm{e}}\right) .
\end{aligned}
$$

Together with Eqs. (6.18) - 6.20) these results lead to the third- and fourth-order predictions

$$
\begin{aligned}
c_{\mathrm{DY}, 3}(x)= & \left.c_{\mathrm{DY}, 3}\right|_{\mathcal{D}_{k}, \delta(1-x)}-512 C_{F}^{3} L_{x}^{5}+\left\{1728 C_{F}^{3}+\frac{640}{3} C_{F}^{2} \beta_{0}\right\} L_{x}^{4} \\
+ & \left\{\left[2272+3072 \zeta_{2}\right] C_{F}^{3}-\left[\frac{544}{3}-512 \zeta_{2}\right] C_{F}^{2} C_{A}\right. \\
& \left.-\left[\frac{2944}{3}+\frac{\xi_{\mathrm{DY}_{3}}}{3}\right] C_{F}^{2} \beta_{0}-\frac{64}{3} C_{F} \beta_{0}^{2}\right\} L_{x}^{3}+O\left(L_{x}^{2}\right)
\end{aligned}
$$

and

$$
\begin{aligned}
c_{\mathrm{DY}, 4}(x)= & \left.c_{\mathrm{DY}, 4}\right|_{\mathcal{D}_{k}, \delta(1-x)}-\frac{4096}{3} C_{F}^{4} L_{x}^{7}+\left\{\frac{19712}{3} C_{F}^{4}+\frac{3584}{3} C_{F}^{3} \beta_{0}\right\} L_{x}^{6} \\
+ & \left\{\left[9088+20480 \zeta_{2}\right] C_{F}^{4}-\left[1408-3072 \zeta_{2}\right] C_{F}^{3} C_{A}\right. \\
& \left.-\left[\frac{20864}{3}+\frac{8 \xi_{\mathrm{DY}_{3}}}{3}\right] C_{F}^{3} \beta_{0}-\frac{1024}{3} C_{F}^{2} \beta_{0}^{2}\right\} L_{x}^{5}+O\left(L_{x}^{4}\right),
\end{aligned}
$$

where the respective third logarithms depend on the presently unknown quantity $\xi_{\mathrm{DY}_{3}}$. Also in Eqs. (6.24) and (6.25) the coefficients of the highest +-distributions and powers of $L_{x} \equiv \ln (1-x)$ for each colour factor are equal in magnitude but opposite in sign. 
Finally we provide the generalizations of Eqs. 3.15 - 3.25) to the next order in $\alpha_{s}$. For the fragmentation functions (2.10) these are given by

$$
\begin{aligned}
& c_{T, 4}(x)=c_{1,4}(x)+\ln ^{6}(1-x) C_{F}^{4}\left\{32 p_{q q}(x) \mathrm{H}_{0}-8+4(1-x)\right\} \\
& +\ln ^{5}(1-x)\left[C _ { F } ^ { 4 } \left\{p_{q q}(x)\left(16 \widetilde{\mathrm{H}}_{1,0}-152 \mathrm{H}_{0,0}-108 \mathrm{H}_{0}+48 \zeta_{2}\right)\right.\right. \\
& \left.+44+176 \mathrm{H}_{0,0}+24 \mathrm{H}_{0}-(1-x)\left(22+88 \mathrm{H}_{0,0}+68 \mathrm{H}_{0}\right)\right\} \\
& +C_{F}^{3} \beta_{0}\left\{-428 / 9 p_{q q}(x) \mathrm{H}_{0}+20-10(1-x)\right\} \\
& \left.+C_{F}^{3} C_{A}\left\{p_{q q}(x)\left(-16 \widetilde{\mathrm{H}}_{1,0}-8 \mathrm{H}_{0,0}\right)-8+4(1-x)\right\}\right] \\
& +O\left(\ln ^{4}(1-x)\right) \text {. } \\
& c_{L, 4}(x)=\ln ^{6}(1-x) 8 / 3 C_{F}^{4} \\
& +\ln ^{5}(1-x)\left[C_{F}^{4}\left\{-4+8 \mathrm{H}_{0}+8 x\right\}-20 / 3 C_{F}^{3} \beta_{0}-16 C_{F}^{3}\left(C_{A}-2 C_{F}\right)\left(1-\zeta_{2}\right)\right] \\
& +\ln ^{4}(1-x)\left[C_{F}^{4}\left\{4-8 \widetilde{\mathrm{H}}_{1,0}-8 \mathrm{H}_{0,0}-4 \mathrm{H}_{0}-32 \zeta_{2}-x\left(12-16 \mathrm{H}_{0}\right)\right\}\right. \\
& +C_{F}^{3} \beta_{0}\left\{112 / 3-56 / 3 \mathrm{H}_{0}-56 / 3 x\right\} \\
& +C_{F}^{3}\left(C_{A}-2 C_{F}\right)\left\{-48 /\left(5 x^{2}\right)\left(\widetilde{\mathrm{H}}_{-1,0}-\zeta_{2} / 2\right)-48 /(5 x)\left(1-\mathrm{H}_{0}\right)+212 / 5\right. \\
& -16\left[2 \widetilde{\mathrm{H}}_{-1,-1,0}-\widetilde{\mathrm{H}}_{-1,0,0}-2 \widetilde{\mathrm{H}}_{0,-1,0}-\widetilde{\mathrm{H}}_{1,0,0}-\widetilde{\mathrm{H}}_{-1,0}\right]-184 / 5 \mathrm{H}_{0}-40 \zeta_{2} \\
& -24 \zeta_{3}+16 \zeta_{2} \mathrm{H}_{0}+x\left(-152 / 5+32 \widetilde{\mathrm{H}}_{-1,0}-32 \mathrm{H}_{0,0}+16 / 5 \mathrm{H}_{0}+48 \zeta_{2}\right) \\
& \left.-32 / 5 x^{2}\left(1+\mathrm{H}_{0}\right)+32 / 5 x^{3}\left(\widetilde{\mathrm{H}}_{-1,0}-\mathrm{H}_{0,0}+\zeta_{2} / 2\right)\right\} \\
& \left.+6 C_{F}^{2} \beta_{0}^{2}+C_{F}^{2}\left(C_{A}-2 C_{F}\right) \beta_{0} 88 / 3\left(1-\zeta_{2}\right)+C_{F}^{2}\left(C_{A}-2 C_{F}\right)^{2} 32\left(\zeta_{2}-\zeta_{3}\right)\right] \\
& +O\left(\ln ^{3}(1-x)\right) \text {. } \\
& c_{A, 4}(x)=c_{T, 4}(x)-\ln ^{6}(1-x) 8 / 3 C_{F}^{4}(1-x) \\
& +\ln ^{5}(1-x)\left[C_{F}^{4}\left\{-64 \mathrm{H}_{0}+(1-x)\left(36+24 \mathrm{H}_{0}-32 \zeta_{2}\right)\right\}\right. \\
& +20 / 3 C_{F}^{3} \beta_{0}(1-x)+C_{F}^{3} C_{A}\left\{32 \mathrm{H}_{0}-16(1-x)\left(1+\mathrm{H}_{0}-\zeta_{2}\right)\right\} \\
& \left.-C_{F}^{3}\left(C_{A}-2 C_{F}\right) 16 p_{q q}(-x)\left(2 \widetilde{\mathrm{H}}_{-1,0}-\mathrm{H}_{0,0}\right)\right] \\
& +o\left(\ln ^{4}(1-x)\right) \text {. }
\end{aligned}
$$

The corresponding result for the third-order Drell-Yan coefficient function reads

$$
\begin{aligned}
c_{\mathrm{DY}, 3}(x) & =\left(\ln ^{5}(1-x) 192 C_{F}^{3}-80 \ln ^{4}(1-x) C_{F}^{2} \beta_{0}\right) p_{q q}(x) \\
& +\ln ^{4}(1-x)\left[C_{F}^{3}\left\{-648 p_{q q}(x) \mathrm{H}_{0}+384 \mathrm{H}_{0}-192(1-x)\left(2+\mathrm{H}_{0}\right)\right\}\right] \\
& +o\left(\ln ^{3}(1-x)\right) .
\end{aligned}
$$

Unlike Eq. (6.17), the fourth-order generalization of Eqs. (3.27) involves more than one unknown coefficient, hence we have not included the incomplete $\ln ^{3}(1-x)$ contribution in Eq. 6.29). 


\section{Numerical illustrations}

We close by briefly illustrating the numerical size of the known and new subleading large- $N$ contributions to the coefficient functions. For $n_{f}=4$ the corresponding expansions of the two- and threeloop coefficient function for $F_{2}$, the practically most important structure function, are given by

$$
\begin{aligned}
c_{2,2}(N)= & 3.556 L^{4}+26.28 L^{3}+40.76 L^{2}-67.13 L-157.3 \\
& +N^{-1}\left(7.111 L^{3}+92.76 L^{2}+239.5 L+214.0\right)+O\left(N^{-2}\right), \\
c_{2,3}(N)= & 3.160 L^{6}+44.92 L^{5}+238.9 L^{4}+470.8 L^{3}-620.2 L^{2}-1639 L-3586 \\
& +N^{-1}\left(9.481 L^{5}+211.9 L^{4}+1393 L^{3}+4157 L^{2}+5200 L+5230\right) \\
& +O\left(N^{-2}\right) .
\end{aligned}
$$

In Fig. 1 these approximations, with and without the $1 / N$ terms, are compared to the exact results of Refs. [23,24] and [27]. At both orders the latter contributions are relevant over the full range of $N$ shown in the figure, while terms of order $1 / N^{2}$ are sizeable only at $N<5$. Note that the classification as $N^{0}$ and $N^{-1}$ terms does not reflect the numerical behaviour for the $N$-values of the figure. E.g., the third-order increase due to the $\ln ^{k} N$ contributions in the first line of Eq. (7.1) strongly resembles a linear rise, and the sum of the $N^{-1} \ln ^{k} N$ terms in the second line almost looks like a constant. In fact, the decrease of this contribution towards large $N$ is very slow: only at $N=1.5 \cdot 10^{2}$ has it fallen to half of the value at its maximum at $N=6.6$. The situation for the corresponding third-order coefficient functions for $F_{1}$ and $F_{3}$ [28], not shown here for brevity, is similar except at small $N$ where in both cases the sum of the $N^{0}$ and $N^{-1}$ terms is close to the exact result even down to $N=1$, the lowest value of $N$ used in the figures.

The pattern of the coefficients is rather different for both the $N^{0}$ and $N^{-1}$ contributions to the corresponding coefficient functions for the transverse fragmentation function $F_{T}$,

$$
\begin{aligned}
c_{T, 2}(N)= & 3.556 L^{4}+25.69 L^{3}+105.6 L^{2}+104.3 L \\
& +N^{-1}\left(7.111 L^{4}-29.02 L^{4}-111.4 L-504.0\right)+o\left(N^{-2}\right), \\
c_{T, 3}(N)= & 3.160 L^{6}+43.34 L^{5}+309.3 L^{4}+1017 L^{3}+2306 L^{2}+2090 L+9332 \\
& +N^{-1}\left(9.481 L^{5}-10.17 L^{4}-362.7 L^{3}-3247 L^{2}\right)+O\left(N^{-1} L\right) .
\end{aligned}
$$

These expansions are shown in Fig. 2 together with the exact second-order result of Refs. [29, 30]. As adequate for an observable measured in particular at scales not too far from the $Z$-mass, the results refer to $n_{f}=5$ effectively light flavours. All $N^{0}$ contributions are positive in Eqs. (7.3) and (7.4), yielding a larger soft-gluon enhancement than in the DIS case especially due to the lower powers of $\ln N$ as already discussed in Ref. [17]. On the other hand, the $N^{-1} \ln ^{k} N$ coefficients change sign here, again in contrast to Eqs. (7.1) and (7.2). This leads to smaller and negative $1 / N$ corrections which do not exceed $10 \%$ except for $N<7$ in the two-loop case in the left part of the figure, where their inclusion results in a good approximation down to $N \simeq 2$. At the third order the $N^{-1} \ln N$ and $N^{-1}$ contributions are not yet known. One may expect similarly relevant small- $N$ corrections from these terms to the corresponding curve shown in the right part of the figure. Similar results are found for the integrated and asymmetric fragmentation functions. 

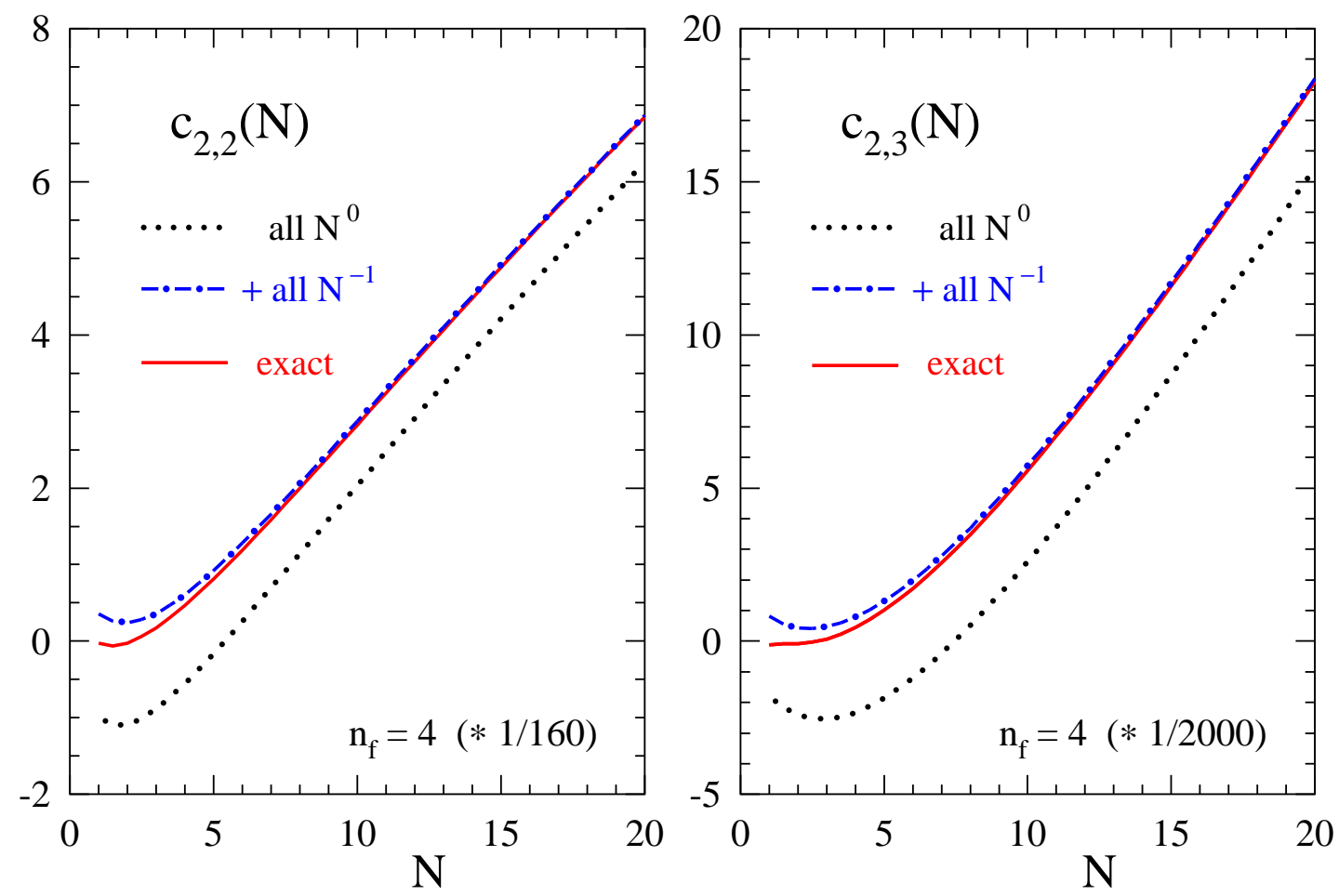

Figure 1: The second- and third-order non-singlet coefficient functions for the structure function $F_{2}$ as defined in Eq. (2.2) in Mellin- $N$ space. The leading and subleading large- $N$ contributions (7.1) and (7.2) are compared to the exact functions for $n_{f}=4$ light flavours. The results are multiplied by suitable factors compensating our small choice $a_{\mathrm{s}}=\alpha_{\mathrm{s}} /(4 \pi)$ of the expansion parameter.
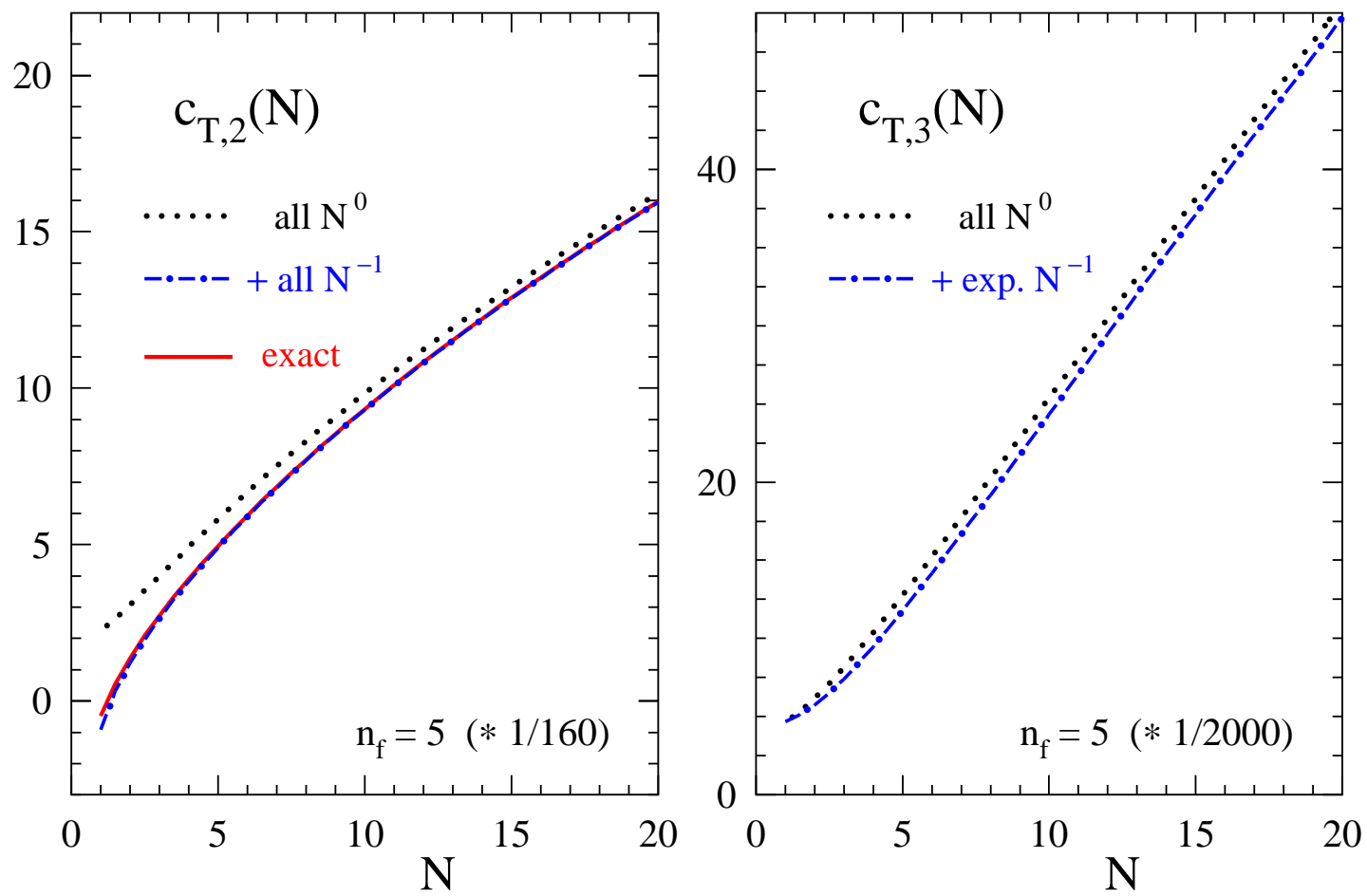

Figure 2: As Fig. 1, but for the fragmentation function $F_{T}$ at $n_{f}=5$. Neither the exact three-loop result nor the corresponding coefficients of the $N^{-1} \ln N$ and $N^{-1}$ terms are known at present. 
We now turn to the four-loop predictions derived in the previous two sections, again focusing on the same two DIS and SIA observables. The known and new contributions at this order read

$$
\begin{aligned}
c_{2,4}(N)= & 2.107 L^{8}+48,71 L^{7}+477.9 L^{6}+2429 L^{5}+5240 L^{4}-1824 L^{3}-30308 L^{2} \\
& +O(L)+N^{-1}\left(8.428 L^{7}+284.3 L^{6}+3324 L^{5}+\left[18884+30.86 \xi_{\mathrm{DIS}_{4}}\right] L^{4}\right) \\
& +O\left(N^{-1} L^{3}\right)
\end{aligned}
$$

and

$$
\begin{aligned}
c_{T, 4}(N)= & 2.107 L^{8}+46.60 L^{7}+514.1 L^{6}+3126 L^{5}+11774 L^{4}+23741 L^{3}+46637 L^{2} \\
& +O(L)+N^{-1}\left(8.428 L^{7}+32.47 L^{6}-448.1 L^{5}-\left[7315+26.12 \xi_{\mathrm{SIA}_{4}}\right] L^{4}\right) \\
& +O\left(N^{-1} L^{3}\right) .
\end{aligned}
$$

As above, the results for $F_{2}$ in Eq. (7.5) are given for $n_{f}=4$, and those for $F_{T}$ in Eq. (7.6) for $n_{f}=5$. The $N^{0}$ coefficients have been presented already in Tables 1 of Refs. [12,17]. The $\ln ^{2} N$ term in both equations includes a small contribution $A_{4} / 2$ from the fourth-order cusp anomalous dimension for which we have used the respective Padé estimates of 4310 for $n_{f}=4$ and 1550 for $n_{f}=5$ [12]. The fourth $N^{-1}$ logarithms receive small contributions from the presently unknown (and most likely identical) fourth-order coefficients $\xi_{\mathrm{DIS}_{4}}$ and $\xi_{\mathrm{SIA}_{4}}$ of Eqs. (5.24) and (6.1). Values expected from the latter equations contribute less than $2 \%$ to the coefficients of $N^{-1} \ln ^{4} N$.

The presently unknown lower- $k N^{-1} \ln ^{k} N$ terms can be expected to enhance the $1 / N$ effects shown in Fig. 3. Yet already now one can conclude that the pattern of the previous two orders appears to persist to order $\alpha_{\mathrm{s}}^{4}$, e.g., that the $N^{-1}$ contributions are small for $F_{T}$ at least at $N \gtrsim 10$. We stress that this figure does not intend to present the best approximation to dominant $N^{0}$ contributions, but simply illustrates the effect of the known terms as given in Eqs. (7.5) and (7.6). Rough estimates of the missing coefficient of $\ln N$ can be obtained by expanding the soft-gluon exponential (4.1) or (also for the non-logarithmic $N^{0}$ terms) via the Mellin transform of the known seven +-distributions given in Eqs. (5.4) - (5.10) of Ref. [12] - note that there are some typos in the first archive and journal versions of this article - and in Eq. (32) of Ref. [17]. The latter article includes also the $c_{2,4}-c_{T, 4}$ difference of the $\ln N$ and $N^{0}$ coefficients in Eqs. (7.5) and (7.6).

Finally the large- $N$ expansion of the second- and third-order coefficient functions for the nonsinglet (quark-antiquark annihilation) Drell-Yan cross section (2.11) are given by

$$
\begin{aligned}
c_{2,2}(N)= & 56.89 L^{4}+185.9 L^{3}+428.6 L^{2}+267.6 L+442.8 \\
& +N^{-1}\left(113.8 L^{3}+378.4 L^{2}+577.3 L+53.43\right)+O\left(N^{-2}\right), \\
c_{2,3}(N)= & 202.3 L^{6}+1282 L^{5}+4676 L^{4}+8172 L^{3}+11404 L^{2}+6395 L+O(1) \\
+ & +N^{-1}\left(606.8 L^{5}+4267 L^{4}+\left[12164-4.543 \xi_{\mathrm{DY}_{3}}\right] L^{3}\right)+O\left(N^{-1} L^{2}\right)
\end{aligned}
$$

for $n_{f}=5$. These expansions are shown in Fig. 4 together with the exact two-loop results of Refs. [31,32]. The higher-order corrections are much larger in this case than in DIS and SIA. Also here the $1 / N$ contributions appear to be numerically rather unimportant, a feature that appears to persists to even lower values of $N$ than for the fragmentation functions. 

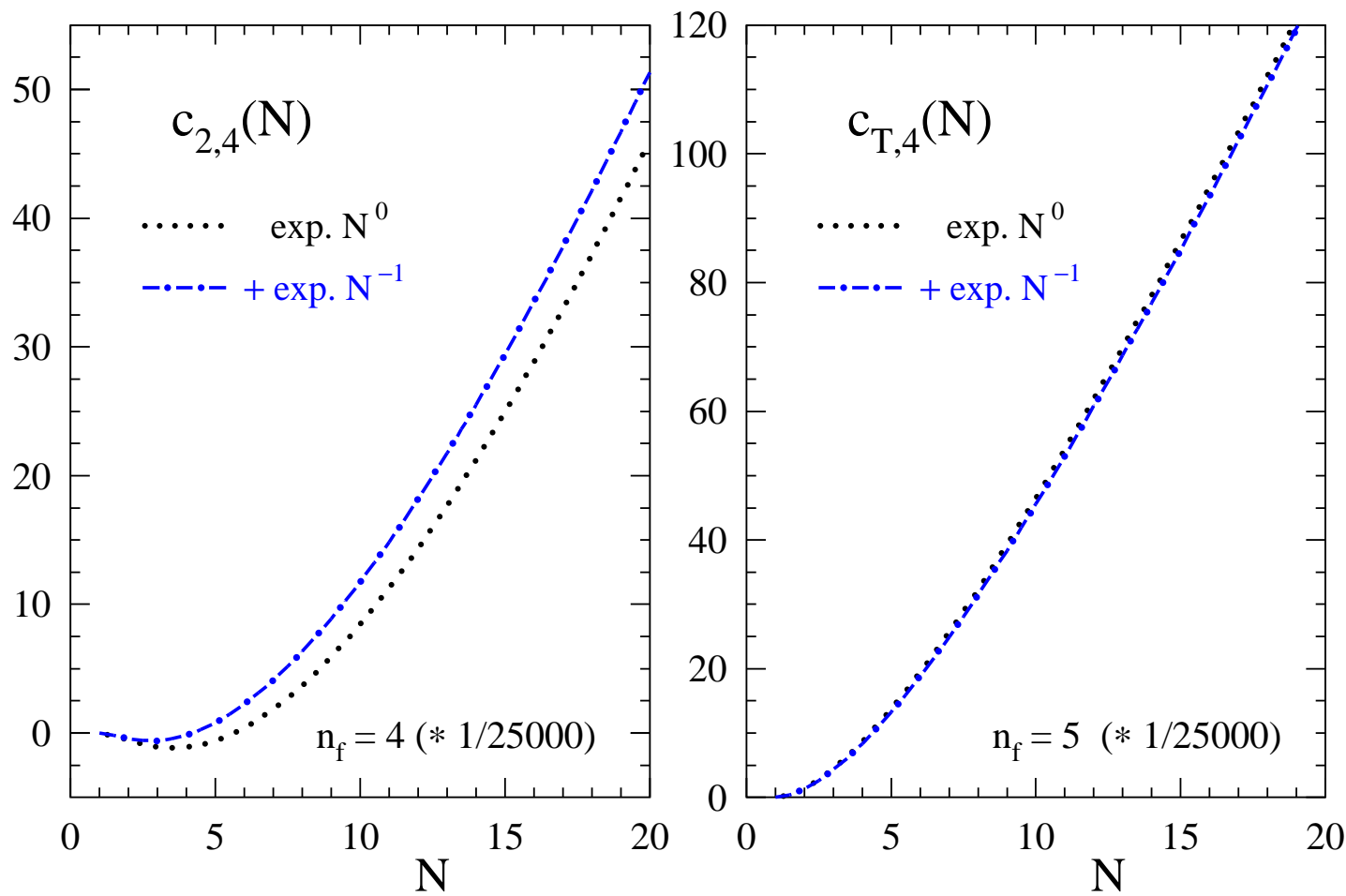

Figure 3: Large- $N$ contributions to the fourth-order non-singlet coefficient functions for $F_{2}$ in DIS (left) and $F_{T}$ in SIA (right). Shown are the known $N^{0}$ and $N^{-1}$ contributions as given in Eqs. (7.5) and Eqs. (7.6). The results have been multiplied by $25000 \simeq(4 \pi)^{4}$ for display purposes.
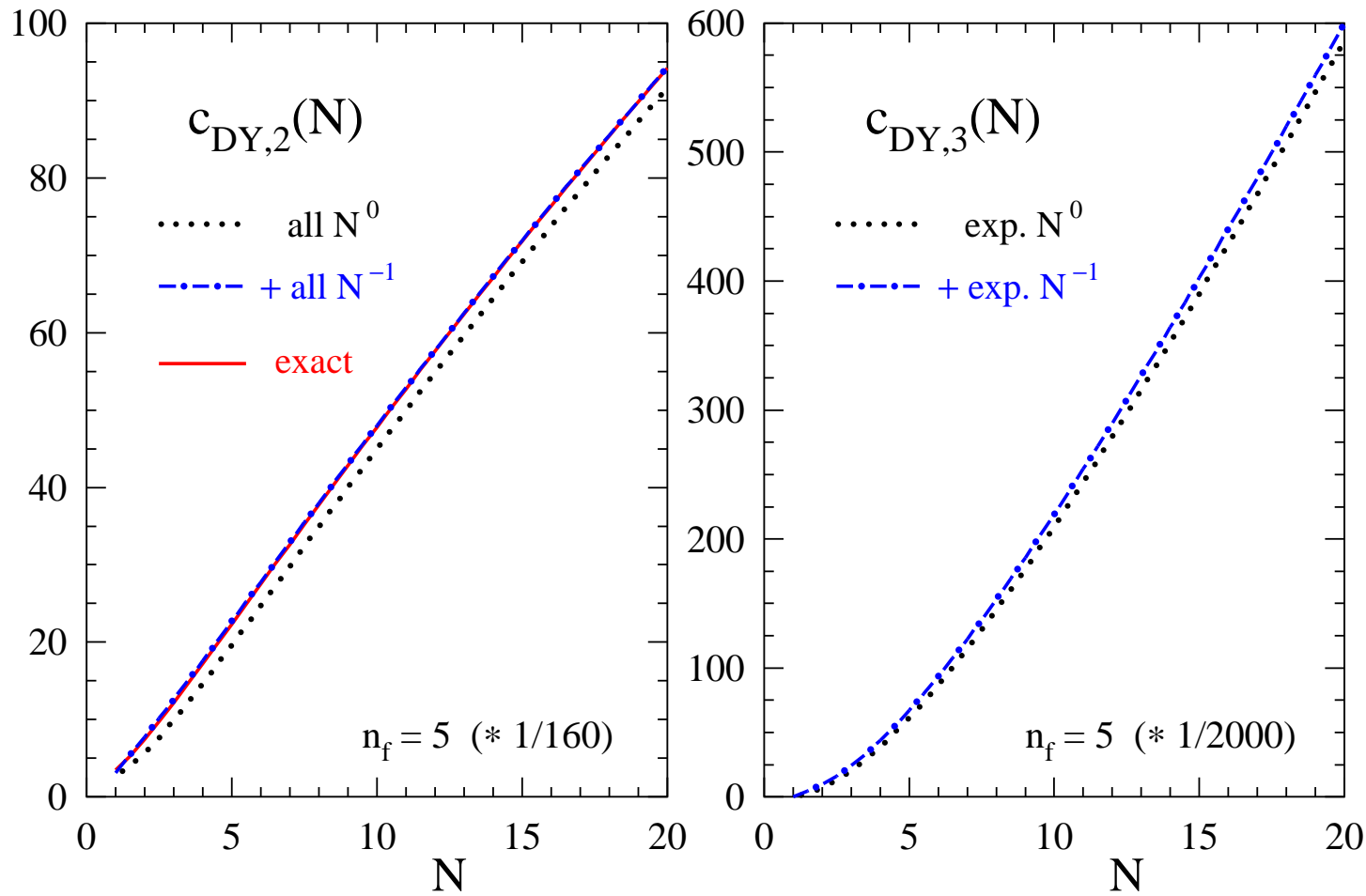

Figure 4: As Fig. 2, but for Drell-Yan cross section (2.11), using the expansions (7.7) and (7.8) with $\xi_{\mathrm{DY}_{3}}=-400$. Besides the coefficients mentioned in the caption of that figure, also the third-order constant- $N$ and $N^{-1} \ln ^{2} N$ coefficient are unknown in this case. 


\section{Summary and outlook}

We have analysed the $\ln ^{k}(1-x)$ contributions to the physical evolution kernels for - including the results already presented in Ref. [34] - nine flavour non-singlet observables in inclusive DIS, semiinclusive $e^{+} e^{-}$annihilation (SIA) and Drell-Yan lepton-pair production. It turns out that all these kernels include only single-logarithmic higher-order corrections, up to $\alpha_{\mathrm{s}}^{n}(1-x)^{k} \ln ^{n-1}(1-x)$, at all powers $k$ of $(1-x)$. On the other hand, the coefficient functions from which these kernels are constructed received double-logarithmic contributions up to $\alpha_{\mathrm{s}}^{n}(1-x)^{k} \ln ^{2 n-1}(1-x)$ at all orders. This difference implies that the terms $\alpha_{\mathrm{s}}^{n}(1-x)^{k} \ln ^{l}(1-x)$ with $n \leq l<2 n$ are functions of lowerorder terms, i.e., a general resummation of the double-logarithmic terms at all powers of $(1-x)$.

The above pattern is established to all order in $\alpha_{\mathrm{s}}$ by the soft-gluon exponentiation of the $(1-x)^{-1} \ln ^{k}(1-x)$ contributions to the coefficient functions [11-17]. All-order results underpinning it at all powers in $(1-x)$ are presently known only for the leading large- $n_{f}$ contributions to DIS structure functions $[35,36]$. However, all available fixed-order results on higher-order coefficient functions [23-32] are consistent with the behaviour described in the previous paragraph.

In our view it is most unlikely that this consistency is accidental, given the large number of observables and the depth of the perturbative expansion reached especially in DIS and SIA - for the latter this article includes some new third-order results. Moreover it should be noted that the resummation of $F_{L}$ in both DIS and SIA can be consistently constructed each via two different physical kernels: that for these quantities themselves (starting with $(1-x)^{-1}$ ) and via the difference (starting with $(1-x)^{0}$ ) of the respective kernels, $K_{2}-K_{1}$ and $K_{I}-K_{T}$, for the structure functions $F_{1,2}$ and fragmentation functions $F_{T, I}$ where $F_{I}$ is our notation for the total (angle-integrated) fragmentation function. We thus definitely expect that we are observing a genuine feature of the coefficient functions and expect that a more deductive approach, such as that pursued in Ref. [19], can provide a formal proof in the near future at least for the next power in $(1-x)$.

We have employed the conjectured single-logarithmic enhancement of the physical kernels to derive the explicit $x$-dependence of the coefficients of the three highest powers of the fourth-order DIS and SIA coefficient functions, while in the Drell-Yan case we are restricted to two logarithms at order $\alpha_{\mathrm{s}}^{3}$. For this purpose we have employed a modified basis (required far beyond the weight-3 functions shown in the article) for the harmonic polylogarithms. An extension of these results to higher orders in $\alpha_{\mathrm{S}}$ is possible but not necessary at present in view of the discussion given below.

For the subdominant (except for $F_{L}$ ) logarithms with prefactor $(1-x)^{0}$ we have cast our results in the form of an exponentiation, akin to that of the $(1-x)^{-1}$ soft-gluon effects, in Mellin- $N$ space where these terms behave as $N^{-1} \ln ^{k} N$. One more logarithm can be effectively predicted in this case, as the one unknown parameter turns out to be numerically suppressed. Our resummation of the $1 / N$ terms is, nevertheless, far less predictive than the soft-gluon exponentiation (which predicts seven of eight fourth-order logarithms in DIS and SIA) for two main reasons: Firstly the prefactor of the exponential is of first instead of zeroth order in $\alpha_{s}$, thus one more order needs to be calculated in order to fix the same number of coefficients. Secondly, while the leading-logarithmic function, usually denoted by $g_{1}\left(\alpha_{\mathrm{S}} \ln N\right)$, in the exponent is the same as in the $N^{0}$ soft-gluon case, this does not hold for the higher-logarithmic functions which have an (at least presently) not fully predictable power expansion (from $g_{2}$ ) and do not show any universality (from $g_{3}$ ). 
Finally we have illustrated the numerical size of the $1 / N$ contributions. It turns out that, in the restricted $N$-region of practical interest, the logarithms at the third and higher orders essentially compensate one power of $N$, i.e., the $N^{0}$ terms together resemble a linear increase with $N$, and $1 / N$ corrections almost look like a constant. The sum of all $N^{0}$ and $N^{-1}$ contributions is found to provide an excellent approximation of the exact results, except at small $N$-values such as $N \lesssim 5$, wherever both are known. However, only in the DIS case do the $1 / N$ terms constitute a phenomelogically significant correction over a wide range of moments.

The main application of the present results and, hopefully, their future extensions in a more deductive approach - we note that also an extension of Ref. [36] to the next-to-leading large$n_{f}$ terms would provide very useful information in the present context - may be in connection with future higher-order diagram calculations, e.g., of the fourth-order DIS coefficient functions: Firstly they can serve as important checks of such computations which will be of unprecedented complexity. Secondly, they will be very useful in combination with future partial results such as

a fourth-order extension of the fixed- $N$ calculations of Refs. [54], as fewer computationally costly moments will be required for useful $x$-space approximations along the lines of, e.g., Ref. [21].

\section{Acknowledgments}

S.M. acknowledges support by the Helmholtz Gemeinschaft under contract VH-NG-105 and in part by the Deutsche Forschungsgemeinschaft in Sonderforschungsbereich/Transregio 9. The research of A.V. has been supported by the UK Science \& Technology Facilities Council (STFC) under grant numbers PP/E007414/1 and ST/G00062X/1.

\section{References}

[1] W.A. Bardeen, A.J. Buras, D.W. Duke, and T. Muta, Phys. Rev. D18 (1978) 3998

[2] G. 't Hooft, Nucl. Phys. B61 (1973) 455

[3] W. Furmanski and R. Petronzio, Z. Phys. C11 (1982) 293

[4] G. 't Hooft and M. Veltman, Nucl. Phys. B44 (1972) 189;

C.G. Bollini and J.J. Giambiagi, Nuovo Cim. 12B (1972) 20;

J.F. Ashmore, Lett. Nuovo Cim. 4 (1972) 289;

G.M. Cicuta and E. Montaldi, Nuovo Cim. Lett. 4 (1972) 329

[5] G. P. Korchemsky, Mod. Phys. Lett. A4 (1989) 1257

[6] S. Moch, J.A.M. Vermaseren and A. Vogt, Nucl. Phys. B688 (2004) 101, hep-ph/0403192

[7] A. Vogt, S. Moch and J.A.M. Vermaseren, Nucl. Phys. B691 (2004) 129, hep-ph/0404111

[8] Y.L. Dokshitzer, G. Marchesini and G.P. Salam, Phys. Lett. B634 (2006) 504, hep-ph/0511302

[9] A. Mitov, S. Moch and A. Vogt, Phys. Lett. B638 (2006) 61, hep-ph/0604053

[10] S. Moch and A. Vogt, Phys. Lett. B659 (2008) 290, arXiv:0709.3899 [hep-ph] 
[11] G. Sterman, Nucl. Phys. B281 (1987) 310; L. Magnea, Nucl. Phys. B349 (1991) 703;

S. Catani and L. Trentadue, Nucl. Phys. B327 (1989) 323; ibid. B353 (1991) 183;

H. Contopanagos, E. Laenen, and G. Sterman, Nucl. Phys. B484 (1997) 303, hep-ph/9604313,

S. Catani, M.L. Mangano, P. Nason and L. Trentadue, Nucl. Phys. B478 (1996) 273, hep-ph/9604351

[12] S. Moch, J.A.M. Vermaseren and A. Vogt, Nucl. Phys. B726 (2005) 317, hep-ph/0506288

[13] S. Moch and A. Vogt, Phys. Lett. B631 (2005) 48, hep-ph/0508265

[14] E. Laenen and L. Magnea, Phys. Lett. B632 (2006) 270, arXiv:hep-ph/0508284

[15] A. Idilbi, X.d. Ji, J.P. Ma and F. Yuan, Phys. Rev. D73 (2006) 077501, hep-ph/0509294

[16] J. Blümlein and V. Ravindran, Phys. Lett. B640 (2006) 40, hep-ph/0605011

[17] S. Moch and A. Vogt, arXiv:0908.2746 [hep-ph], Phys. Lett. B (in press)

[18] G. Grunberg, arXiv:0710.5693 [hep-ph];

E. Laenen, L. Magnea and G. Stavenga, Phys. Lett. B669 (2008) 173, arXiv:0807.4412 [hep-ph];

G. Grunberg and V. Ravindran, arXiv:0902.2702 [hep-ph]

[19] E. Laenen, G. Stavenga and C.D. White, JHEP 0903 (2009) 054 arXiv:0811.2067 [hep-ph]

[20] J. Blümlein, V. Ravindran and W. L. van Neerven, Nucl. Phys. B586, 349 (2000), hep-ph/0004172

[21] W.L. van Neerven and A. Vogt, Nucl. Phys. B603 (2001) 42, hep-ph/0103123

[22] S. Forte and G. Ridolfi, Nucl. Phys. B650 (2003) 229, hep-ph/0209154,

E. Gardi and R. G. Roberts, Nucl. Phys. B653 (2003) 227, hep-ph/0210429

[23] W.L. van Neerven and E.B. Zijlstra, Phys. Lett. B272 (1991) 127;

E.B. Zijlstra and W.L. van Neerven, ibid. B297 (1992) 377

[24] S. Moch and J.A.M. Vermaseren, Nucl. Phys. B573 (2000) 853, hep-ph/9912355

[25] S. Moch, J.A.M. Vermaseren and A. Vogt, Nucl. Phys. B646 (2002) 181, hep-ph/0209100

[26] S. Moch, J.A.M. Vermaseren and A. Vogt, Phys. Lett. B606 (2005) 123, hep-ph/0411112

[27] J.A.M. Vermaseren, A. Vogt and S. Moch, Nucl. Phys. B724 (2005) 3, hep-ph/0504242

[28] S. Moch, J.A.M. Vermaseren and A. Vogt, Nucl. Phys. B813 (2009) 220, arXiv:0812.45176 [hep-ph]

[29] P.J. Rijken and W.L. van Neerven, Phys. Lett. B386 (1996) 422, hep-ph/9604436, Nucl. Phys. B488 (1997) 233, hep-ph/9609377; Phys. Lett. B392 (1997) 207, hep-ph/9609379

[30] A. Mitov and S. Moch, Nucl. Phys. B751 (2006) 18, arXiv:hep-ph/0604160

[31] R. Hamberg, W.L. van Neerven and T. Matsuura, Nucl. Phys. B359 (1991) 343 [Erratum ibid. B644 (2002) 403 ]

[32] R.V. Harlander and W.B. Kilgore, Phys. Rev. Lett. 88 (2002) 201801, hep-ph/0201206

[33] C. Amsler et al., Particle Data Group, Phys. Lett. B667 (2008) 1

[34] S. Moch and A. Vogt, JHEP 0904 (2009) 081, arXiv:0902.2342 [hep-ph]

[35] J.A. Gracey, hep-ph/9509276, Proceedings of the 4th International Workshop on Software Engineering and Artificial Intelligence for High-Energy and Nuclear Physics (AIHENP 95), Pisa, April 1995, eds. B. Denby and D. Perret-Gallix (World Scientific 1995), p. 259

[36] L. Mankiewicz, M. Maul and E. Stein, Phys. Lett. B404 (1997) 345, hep-ph/9703356 
[37] D.J. Gross and F. Wilczek, Phys. Rev. Lett. 30 (1973) 1343;

H.D. Politzer, Phys. Rev. Lett. 30 (1973) 1346

[38] W.E. Caswell, Phys. Rev. Lett. 33 (1974) 244;

D.R.T. Jones, Nucl. Phys. B75 (1974) 531

[39] O.V. Tarasov, A.A. Vladimirov, and A.Yu. Zharkov, Phys. Lett. B93 (1980) 429;

S.A. Larin and J.A.M. Vermaseren, Phys. Lett. B303 (1993) 334

[40] T. van Ritbergen, J.A.M. Vermaseren and S.A. Larin, Phys. Lett. B400 (1997) 379, hep-ph/9701390;

M. Czakon, Nucl. Phys. B710 (2005) 485, hep-ph/0411261

[41] J.A.M. Vermaseren, Int. J. Mod. Phys. A14 (1999) 2037, hep-ph/9806280,

J. Blümlein and S. Kurth, Phys. Rev. D60 (1999) 014018, hep-ph/9810241

[42] J.A.M. Vermaseren, math-ph/0010025

[43] M. Tentyukov and J.A.M. Vermaseren, arXiv:hep-ph/0702279

[44] E. Remiddi and J.A.M. Vermaseren, Int. J. Mod. Phys. A15 (2000) 725, hep-ph/9905237

[45] J.A. Gracey, Phys. Lett. B322 (1994) 141, hep-ph/9401214

[46] P.A. Baikov and K.G. Chetyrkin, Nucl. Phys. B (Proc. Suppl.) 160 (2006) 76

[47] E.G. Floratos, D.A. Ross and C.T. Sachrajda, Nucl. Phys. B129 (1977) 66 [Erratum ibid. B139 (1978) 545]

[48] G. Curci, W. Furmanski and R. Petronzio, Nucl. Phys. B175 (1980) 27

[49] B. Basso and G. P. Korchemsky, Nucl. Phys. B775 (2007) 1, hep-th/0612247

[50] M. Krämer, E. Laenen and M. Spira, Nucl. Phys. B511 (1998) 523

[51] A. Vogt, Phys. Lett. B471 (1999) 97, hep-ph/9910545

[52] J. Kodaira and L. Trentadue, Phys. Lett. 112B (1982) 66

[53] A. Vogt, Phys. Lett. B497 (2001) 228, hep-ph/0010146

[54] S.A. Larin, T. van Ritbergen and J.A.M. Vermaseren, Nucl. Phys. B427 (1994) 41;

S.A. Larin, P. Nogueira, T. van Ritbergen and J. Vermaseren, Nucl. Phys. B492 (1997) 338, hep-ph/9605317,

A. Retey and J.A.M. Vermaseren, Nucl. Phys. B604 (2001) 281, hep-ph/0007294 